\title{
Automorphisms of the plane preserving a curve
}

\author{
Jérémy Blanc and Immanuel Stampfli
}

\begin{abstract}
We study the group of automorphisms of the affine plane preserving some given curve, over any field. The group is proven to be algebraic, except in the case where the curve is a union of parallel lines. Moreover, a classification of the groups of positive dimension occurring is also given in the case where the curve is geometrically irreducible and the field is perfect.
\end{abstract}

\section{Introduction}

Let $\mathbf{k}$ be an arbitrary field. This article studies (closed) curves $\Gamma \subset \mathbb{A}^{2}=\mathbb{A}_{\mathbf{k}}^{2}$ and the group of automorphisms of $\mathbb{A}^{2}$ (defined over $\mathbf{k}$ ) which preserve this curve. We will denote this group by $\operatorname{Aut}\left(\mathbb{A}^{2}, \Gamma\right)$. In other words, we study polynomials in $\mathbf{k}[x, y]$ and the $\mathbf{k}$-algebra automorphisms of $\mathbf{k}[x, y]$ that send the polynomial to a multiple of itself. We will always assume that the curve is reduced, that is, that the polynomial does not contain any multiple factor. For our purpose, this is a natural assumption.

If $\Gamma$ has equation in $\mathbf{k}[x]$, we will say that $\Gamma$ is a fence. In this case, $\operatorname{Aut}\left(\mathbb{A}^{2}, \Gamma\right)$ is easy to describe, and it is in fact a countable union of algebraic groups. Our main result consists in showing that this is the only case where such a phenomenon occurs.

Recall that $\operatorname{Aut}\left(\mathbb{A}^{2}\right)$ has the structure of an ind-variety. More precisely, the set $\operatorname{Aut}\left(\mathbb{A}^{2}\right)_{d}$ of automorphisms of degree at most $d$ is an algebraic variety and $\operatorname{Aut}\left(\mathbb{A}^{2}\right)_{d}$ is closed in $\operatorname{Aut}\left(\mathbb{A}^{2}\right)_{d+1}$ for any $d$. This gives to $\operatorname{Aut}\left(\mathbb{A}^{2}\right)=\bigcup_{d=1}^{\infty} \operatorname{Aut}\left(\mathbb{A}^{2}\right)_{d}$ the structure of an ind-variety, and since composition and taking inverse preserve this structure, Aut $\left(\mathbb{A}^{2}\right)$ is an ind-group (see $[\mathrm{Kum02}$, Chapter IV] for precise definitions of "ind-variety" and "ind-group").

By definition, an algebraic subgroup of $\operatorname{Aut}\left(\mathbb{A}^{2}\right)$ is a closed subgroup of bounded degree. Let us recall that the group $\operatorname{Aut}\left(\mathbb{A}^{2}\right)$ contains the following natural algebraic subgroups:

$$
\begin{aligned}
\operatorname{Aff}\left(\mathbb{A}^{2}\right) & =\{(x, y) \mapsto(a x+b y+e, c x+d y+f) \mid a, b, c, d, e, f \in \mathbf{k}, a d-b c \neq 0\}, \\
\mathrm{J}_{n} & =\left\{(x, y) \mapsto(a x+P(y), b y+c) \mid a, b \in \mathbf{k}^{*}, c \in \mathbf{k}, P \in \mathbf{k}[y], \operatorname{deg}(P) \leqslant n\right\} .
\end{aligned}
$$

Moreover, Aut $\left(\mathbb{A}^{2}\right)$ is generated by the union of these groups (Jung-van der Kulk's theorem [Jun42], [vdK53]), and any algebraic subgroup of $\operatorname{Aut}\left(\mathbb{A}^{2}\right)$ is conjugate to a subgroup of $\operatorname{Aff}\left(\mathbb{A}^{2}\right)$ or $\mathrm{J}_{n}$ for some $n$ [Kam79, Theorem 4.3].

Received 16 April 2013, accepted in final form 31 July 2014.

2010 Mathematics Subject Classification 14R10, 14R20, 14H37, 14H50, 14J50, 14E07

Keywords: plane algebraic curves, birational geometry of surfaces, affine automorphisms

This journal is (C) Foundation Compositio Mathematica 2015. This article is distributed with Open Access under the terms of the Creative Commons Attribution Non-Commercial License, which permits non-commercial reuse, distribution, and reproduction in any medium, provided that the original work is properly cited. For commercial re-use, please contact the Foundation Compositio Mathematica.

The authors gratefully acknowledge support by the Swiss National Science Foundation Grants "Birational Geometry" 128422 and "Automorphisms of Affine n-Space" 137679. 


\section{JÉRÉMy BlanC AND IMMANUEl StAMPfli}

For any curve $\Gamma \subset \mathbb{A}^{2}$, the group $\operatorname{Aut}\left(\mathbb{A}^{2}, \Gamma\right)$ is a closed ind-subgroup of $\operatorname{Aut}\left(\mathbb{A}^{2}\right)$. The following result describes when $\operatorname{Aut}\left(\mathbb{A}^{2}, \Gamma\right)$ is an algebraic group, that is, when it has bounded degree.

TheOrem 1. Let $\Gamma$ be a curve in $\mathbb{A}^{2}$. Applying an automorphism of $\mathbb{A}^{2}$, one of the following holds:

(i) The curve $\Gamma$ has equation $F(x)=0$, where $F(x) \in \mathbf{k}[x]$ is a square-free polynomial and

$$
\begin{aligned}
\operatorname{Aut}\left(\mathbb{A}^{2}, \Gamma\right)= & \left\{(x, y) \mapsto(a x+b, c y+P(x)) \mid a, c \in \mathbf{k}^{*}, b \in \mathbf{k},\right. \\
& \left.P \in \mathbf{k}[x], F(a x+b) / F(x) \in \mathbf{k}^{*}\right\} .
\end{aligned}
$$

(ii) The group $\operatorname{Aut}\left(\mathbb{A}^{2}, \Gamma\right)$ is equal to

$$
\left\{g \in \operatorname{Aff}\left(\mathbb{A}^{2}\right) \mid g(\Gamma)=\Gamma\right\} \quad \text { or } \quad\left\{g \in \mathrm{J}_{n} \mid g(\Gamma)=\Gamma\right\}
$$

for some integer $n$. Moreover, the action of $\operatorname{Aut}\left(\mathbb{A}^{2}, \Gamma\right)$ on $\Gamma$ gives an isomorphism of $\operatorname{Aut}\left(\mathbb{A}^{2}, \Gamma\right)$ with a closed subgroup of $\operatorname{Aut}(\Gamma)$ (the latter being an algebraic group).

In particular, Aut $\left(\mathbb{A}^{2}, \Gamma\right)$ is an algebraic group if and only if there is no automorphism of $\mathbb{A}^{2}$ which sends $\Gamma$ onto a fence.

Note that the existence of an automorphism of $\mathbb{A}^{2}$ which sends $\Gamma$ onto a line implies that $\Gamma \simeq \mathbb{A}^{1}$, and that the converse is true in characteristic zero by the Abhyankar-Moh-Suzuki theorem ([Suz74], [AM75]), but false in general (last page of [Nag71]). So Theorem 1 implies, in positive characteristic, that non-trivial embeddings of $\mathbb{A}^{1}$ into $\mathbb{A}^{2}$ are rigid in the sense that the image of the curve is preserved by only a few automorphisms of $\mathbb{A}^{2}$, namely an algebraic group.

Another consequence of Theorem 1 is that the fixed locus of an automorphism of $\mathbb{A}^{2}$ contains only points and curves equivalent to lines, a result already observed by Friedland and Milnor in [FM89], in the case where $\mathbf{k}=\mathbb{C}$ (see [Jel03] for some generalisations to higher dimensions).

Note that in the case where $\mathbf{k}=\mathbb{C}$, the observation on fixed points of Friedland and Milnor, the Abhyankar-Moh-Suzuki theorem and the Lin-Zaidenberg theorem [ZL83] imply Theorem 1 in the case where the curve $\Gamma$ has an irreducible component non-isomorphic to $\mathbb{C}^{*}$. The interesting case of Theorem 1 , for $\mathbf{k}=\mathbb{C}$, is thus the description of $\operatorname{Aut}\left(\mathbb{A}^{2}, \Gamma\right)$ when $\Gamma$ is isomorphic to $\mathbb{C}^{*}$ (or a union of such curves). Note that there exist only partial classifications of the closed embeddings of $\mathbb{C}^{*}$ into $\mathbb{A}^{2}$, which are moreover very involved (see [CNKR09], [BŻa10] and more recently [KPR14]). Hence, one cannot check a few cases to derive Theorem 1. Moreover, note that there exist complicated torsion-abelian closed subgroups of $\operatorname{Aut}\left(\mathbb{A}^{2}\right)$ which are not conjugate to a subgroup of $\operatorname{Aff}\left(\mathbb{A}^{2}\right)$ or to a subgroup of the de Jonquières group $\bigcup_{n=1}^{\infty} \mathrm{J}_{n}$ (see [Wri79]). A priori, such groups could preserve curves isomorphic to $\mathbb{A}^{1} \backslash\{0\}$, but Theorem 1 implies that these groups do not preserve any algebraic curves.

The proof of Theorem 1 is given in Section 3, using tools of birational geometry of surfaces introduced in Section 2.

In Section 4, we refine the theorem by describing more precisely the possibilities for the group $\operatorname{Aut}\left(\mathbb{A}^{2}, \Gamma\right)$ in the case where $\Gamma$ is geometrically irreducible and the ground field $\mathbf{k}$ is perfect. We then obtain the following result (the tori $T$ of cases (iv), (v) are described precisely in Proposition 4.5).

THEOREM 2. Let $\Gamma$ be a geometrically irreducible closed curve in $\mathbb{A}^{2}$, defined over a perfect field $\mathbf{k}$. Applying an automorphism of $\mathbb{A}^{2}$, one of the following holds:

(i) The curve $\Gamma$ is the line with equation $x=0$ and

$$
\operatorname{Aut}\left(\mathbb{A}^{2}, \Gamma\right)=\left\{(x, y) \mapsto(a x, b y+P(x)) \mid a, b \in \mathbf{k}^{*}, P \in \mathbf{k}[x]\right\} .
$$




\section{Automorphisms of the Plane PRESERVING A CURVE}

(ii) The curve $\Gamma$ has equation $x^{b}=\lambda y^{a}$, where $\lambda \in \mathbf{k}^{*}$ and $a, b>1$ are coprime integers. Moreover, Aut $\left(\mathbb{A}^{2}, \Gamma\right)$ is equal to the group $\mathbf{k}^{*}$ acting diagonally via $(x, y) \mapsto\left(t^{a} x, t^{b} y\right)$.

(iii) The curve $\Gamma$ has equation $x^{b} y^{a}=\lambda$, where $\lambda \in \mathbf{k}^{*}$ and $a, b \geqslant 1$ are coprime integers. Moreover, Aut $\left(\mathbb{A}^{2}, \Gamma\right)$ contains the group $\mathbf{k}^{*}$ acting diagonally via $(x, y) \mapsto\left(t^{a} x, t^{-b} y\right)$, and is equal to this group if $(a, b) \neq(1,1)$, or is the group $\mathbf{k}^{*} \rtimes \mathbb{Z} / 2 \mathbb{Z}$ generated by $\mathbf{k}^{*}$ and by $(x, y) \mapsto(y, x)$ if $(a, b)=(1,1)$.

(iv) The curve $\Gamma$ has equation $\lambda x^{2}+\nu y^{2}=1$, where $\lambda, \nu \in \mathbf{k}^{*},-\lambda \nu$ is not a square in $\mathbf{k}$ and $\operatorname{char}(\mathbf{k}) \neq 2$. The group Aut $\left(\mathbb{A}^{2}, \Gamma\right)$ is the subgroup of $\operatorname{GL}(2, \mathbf{k})$ preserving the form $\lambda x^{2}+\nu y^{2}$. It is isomorphic to $T \rtimes \mathbb{Z} / 2 \mathbb{Z}$ for some non-k-split torus $T$.

(v) The curve $\Gamma$ has equation $x^{2}+\mu x y+y^{2}=1$, where $\mu \in \mathbf{k}^{*}$ and $x^{2}+\mu x+1$ has no root in $\mathbf{k}$, and $\operatorname{char}(\mathbf{k})=2$. The group $\operatorname{Aut}\left(\mathbb{A}^{2}, \Gamma\right)$ is the subgroup of $\mathrm{GL}(2, \mathbf{k})$ preserving the form $x^{2}+\mu x y+y^{2}$. It is isomorphic to $T \rtimes \mathbb{Z} / 2 \mathbb{Z}$ for some non-k-split torus $T$.

(vi) The group $\operatorname{Aut}\left(\mathbb{A}^{2}, \Gamma\right)$ is a zero-dimensional (hence finite) subgroup of $\operatorname{Aff}\left(\mathbb{A}^{2}\right)$ or $\mathrm{J}_{n}$ for some $n$.

A similar description was also given in the case $\mathbf{k}=\mathbb{C}$ and where the curve is connected and simply connected in [AZ13], and more generally for such curves on affine toric surfaces. The intersection of the two studies gives the cases (i) and (ii) above.

\section{Reminders on completions of $\mathbb{A}^{2}$}

In this section, we define natural completions of $\mathbb{A}^{2}$ and links between them.

\subsection{Natural completions of $\mathbb{A}^{2}$}

EXAMPLE 2.1. The morphism

$$
\begin{aligned}
\mathbb{A}^{2} & \hookrightarrow \mathbb{P}^{2} \\
(x, y) & \mapsto(x: y: 1)
\end{aligned}
$$

yields an isomorphism $\mathbb{A}^{2} \stackrel{\sim}{\rightarrow} \mathbb{P}^{2} \backslash L_{\mathbb{P}^{2}}$, where $L_{\mathbb{P}^{2}}$ is the line of $\mathbb{P}^{2}$ with equation $z=0$.

EXAmPLE 2.2. For $n \geqslant 1$, the $n$th Hirzebruch surface $\mathbb{F}_{n}$ is

$$
\mathbb{F}_{n}=\left\{((a: b: c),(u: v)) \in \mathbb{P}^{2} \times \mathbb{P}^{1} \mid b v^{n}=c u^{n}\right\} .
$$

Let $E_{n}, L_{\mathbb{F}_{n}} \subset \mathbb{F}_{n}$ be the curves given by $(1: 0: 0) \times \mathbb{P}^{1}$ and $v=0$, respectively. The morphism

$$
\begin{aligned}
\mathbb{A}^{2} & \hookrightarrow \mathbb{F}_{n} \\
(x, y) & \mapsto\left(\left(x: y^{n}: 1\right),(y: 1)\right)
\end{aligned}
$$

gives an isomorphism $\mathbb{A}^{2} \stackrel{\sim}{\rightarrow} \mathbb{F}_{n} \backslash\left(E_{n} \cup L_{\mathbb{F}_{n}}\right)$. Note that $E_{n}$ is the unique section of $\pi: \mathbb{F}_{n} \rightarrow \mathbb{P}^{1}$ of self-intersection $-n$, and $L_{\mathbb{F}_{n}}$ has self-intersection zero since it is a smooth fibre.

Remark 2.3. In Example 2.2, we could also have chosen $n=0$, which yields the surface $\mathbb{F}_{0}$, isomorphic to $\mathbb{P}^{1} \times \mathbb{P}^{1}$, via $((x: y: z),(u: v)) \mapsto((x: y),(u: v))$, but we will not need this case.

Definition 2.4. A natural completion (of $\mathbb{A}^{2}$ ) is a pair $(X, B)$ which is given in Example 2.1 or Example 2.2: either $(X, B)=\left(\mathbb{P}^{2}, L_{\mathbb{P}^{2}}\right)$ or $(X, B)=\left(\mathbb{F}_{n}, E_{n} \cup L_{\mathbb{F}_{n}}\right)$ for some $n \geqslant 1$. The isomorphism $\mathbb{A}^{2} \rightarrow X \backslash B$ given in the examples will be called the canonical isomorphism, or canonical embedding of $\mathbb{A}^{2}$ into $X$. 


\section{JÉRÉMy BLANC AND IMMANUEl StAMPfli}

A birational map (respectively, birational morphism) $(X, B) \rightarrow\left(X^{\prime}, B^{\prime}\right)$ between two natural completions is a birational map (respectively, birational morphism) $X \rightarrow X^{\prime}$ inducing an isomorphism $X \backslash B \rightarrow X^{\prime} \backslash B^{\prime}$.

We denote by $\operatorname{Aut}(X, B)$ the group of automorphisms of $(X, B)$; it is the group of automorphisms of $X$ which leave $B$ (or, equivalently, $X \backslash B=\mathbb{A}^{2}$ ) invariant.

Remark 2.5. Given two natural completions $(X, B),\left(X^{\prime}, B^{\prime}\right)$, any birational map $\varphi:(X, B)--\rightarrow$ $\left(X^{\prime}, B^{\prime}\right)$ restricts to an isomorphism $X \backslash B \rightarrow X^{\prime} \backslash B^{\prime}$, and thus corresponds, via the canonical isomorphisms $\mathbb{A}^{2} \simeq X \backslash B$ and $\mathbb{A}^{2} \simeq X^{\prime} \backslash B^{\prime}$, to a unique automorphism of $\mathbb{A}^{2}$. Moreover, every automorphism of $\mathbb{A}^{2}$ is obtained in this way.

Let us recall an easy fact on the automorphisms of natural completions.

Lemma 2.6. Let $(X, B)$ be a natural completion of $\mathbb{A}^{2}$, and let $\iota: \mathbb{A}^{2} \rightarrow X \backslash B$ be the associated canonical isomorphism. The group $\operatorname{Aut}(X, B)$ corresponds via $\iota$ to the following subgroups of $\operatorname{Aut}\left(\mathbb{A}^{2}\right)$ (see the introduction for the definition of $\operatorname{Aff}\left(\mathbb{A}^{2}\right)$ and $\left.\mathrm{J}_{n}\right)$ :

(i) If $X=\mathbb{P}^{2}$, then $\operatorname{Aut}(X, B)=\operatorname{Aff}\left(\mathbb{A}^{2}\right) \simeq \mathrm{GL}(2, \mathbf{k}) \ltimes \mathbf{k}^{2}$.

(ii) If $X=\mathbb{F}_{n}$, then $\operatorname{Aut}(X, B)=\mathrm{J}_{n} \simeq\left(\mathbf{k}^{*}\right)^{2} \ltimes\left(\mathbf{k} \ltimes \mathbf{k}^{n+1}\right)$.

Proof. The first assertion follows from the fact that $\operatorname{Aut}\left(\mathbb{P}^{2}\right)$ is the group of linear automorphisms, equal to $\operatorname{PGL}(3, \mathbf{k})$.

Recall that for $n \geqslant 1$, the $\mathbb{P}^{1}$-bundle structure on the Hirzebruch surface $\mathbb{F}_{n}$ given by the projection on the second factor is unique. Hence, the group of automorphisms of $\mathbb{F}_{n}$ preserves the $\mathbb{P}^{1}$-bundle structure. In particular, the restriction of each automorphism of $\operatorname{Aut}\left(\mathbb{F}_{n}, E_{n} \cup L_{\mathbb{F}_{n}}\right)$ is an automorphism of $\mathbb{A}^{2}$ that preserves the fibration $\mathbb{A}^{2} \rightarrow \mathbb{A}^{1},(x, y) \mapsto y$. The precise description of the degree follows from a straightforward calculation.

\subsection{Elementary links}

There are two kinds of very simple birational maps between natural completions. The first ones are automorphisms, which we described in Lemma 2.6, and the second ones are elementary links, which we describe now. The results of this section are classic, we just recall them for the reader for self-containedness and in order to fix notation.

Definition 2.7. A link is a birational map $\varphi:(X, B) \rightarrow\left(X^{\prime}, B^{\prime}\right)$ between two natural completions, which is not an isomorphism, such that both $\varphi$ and $\varphi^{-1}$ have at most one $\overline{\mathbf{k}}$-base point (and, in particular, there is no infinitely near base point). An elementary link is a link which does not decompose into $\varphi=\varphi^{\prime} \circ \varphi^{\prime \prime}$, where $\varphi^{\prime}$ and $\varphi^{\prime \prime}$ are links.

Remark 2.8. It follows from Definition 2.7 that if $\varphi:(X, B) \rightarrow\left(X^{\prime}, B^{\prime}\right)$ is an elementary link, then so is $\alpha \varphi \beta$, where $\alpha \in \operatorname{Aut}\left(X^{\prime}, B^{\prime}\right), \beta \in \operatorname{Aut}(X, B)$.

The following lemma gives a precise description of our elementary links; this shows, in particular, that such maps are some of the elementary links which appear in the minimal model program (see [Cor95, Theorem on p. 225] and [Isk96, Definition 2.2, p. 597]).

Lemma 2.9. Any elementary link is of one of the following three types:

(i) type I: a rational map $\left(\mathbb{P}^{2}, L_{\mathbb{P}^{2}}\right) \rightarrow\left(\mathbb{F}_{1}, E_{1} \cup L_{\mathbb{F}_{1}}\right)$ consisting of blowing up one $\mathbf{k}$-rational point of $L_{\mathbb{P}^{2}}$; 


\section{Automorphisms of the Plane PRESERVing a CURVE}

(ii) type II: a rational map $\left(\mathbb{F}_{n}, E_{n} \cup L_{\mathbb{F}_{n}}\right) \rightarrow\left(\mathbb{F}_{m}, E_{m} \cup L_{\mathbb{F}_{m}}\right)$ given by the blow-up of a k-rational point $p$ of $L_{\mathbb{F}_{n}}$ followed by the contraction of the strict transform of $L_{\mathbb{F}_{n}}$, where, moreover, $m=n+1$ if $p \in E_{n}$ and $m=n-1$ if $p \notin E_{n}$;

(iii) type III: a morphism $\left(\mathbb{F}_{1}, E_{1} \cup L_{\mathbb{F}_{1}}\right) \rightarrow\left(\mathbb{P}^{2}, L_{\mathbb{P}^{2}}\right)$ given by the contraction of the curve $E_{1}$ onto a k-rational point of $L_{\mathbb{P}^{2}}$.

The inverse of a link of type I, II, III is a link of type III, II, I, respectively.

Proof. Let $\varphi:(X, B) \rightarrow\left(X^{\prime}, B^{\prime}\right)$ be a birational map that is an elementary link.

Suppose first that $\varphi$ is a morphism, which implies that $\varphi$ is the contraction of a $(-1)$-curve onto a k-rational point. Hence, $X=\mathbb{F}_{1}, X^{\prime}=\mathbb{P}^{2}$ and we are in the third case. If $\varphi^{-1}$ is a morphism, we get the first case by symmetry.

In the remaining cases, both $\varphi$ and $\varphi^{-1}$ have exactly one base point. These points are thus defined over $\mathbf{k}$, and both maps contract one irreducible curve. The curves thus have either selfintersection -1 or self-intersection 0 , depending on whether they contain the base points. In the first case, we get a link $\left(\mathbb{F}_{1}, E_{1} \cup L_{\mathbb{F}_{1}}\right) \rightarrow\left(\mathbb{F}_{1}, E_{1} \cup L_{\mathbb{F}_{1}}\right)$, which is not an elementary link since it factors through $\mathbb{P}^{2}$ as the composition of links of types III and I. In the second case, we get a link of type II described above.

Proposition 2.10. Let $\varphi:(X, B) \rightarrow\left(X^{\prime}, B^{\prime}\right)$ be a birational map between two natural completions of $\mathbb{A}^{2}$. If $\varphi$ is not an isomorphism, then there exist an $m \geqslant 1$ and elementary links $\varphi_{1}, \ldots, \varphi_{m}$ such that $\varphi=\varphi_{m} \cdots \varphi_{1}$.

Remark 2.11. We call $\varphi=\varphi_{m} \cdots \varphi_{1}$ a decomposition into $m$ elementary links. If $\varphi$ is an isomorphism, we sometimes say that it decomposes into zero elementary links. This is coherent with the fact that the composition of an elementary link with an isomorphism of natural completions is an elementary link (see Remark 2.8).

Proposition 2.10 implicitly follows from the work done in [Lam02] or from [BD11, Theorem 3.0.2]. However, the proof given here is direct.

Proof of Proposition 2.10. As all birational maps between projective smooth surfaces, $\varphi$ admits a minimal resolution, that is, two birational morphisms $\epsilon: Y \rightarrow X, \eta: Y \rightarrow X^{\prime}$ such that $\varphi=\eta \epsilon^{-1}$, and such that no $(-1)$-curve of $Y$ (not necessarily defined over $\mathbf{k}$ ) is contracted by both $\epsilon$ and $\eta$. Moreover, each point blown up by $\eta$ and $\epsilon$ belongs to the boundaries $B, B^{\prime}$, as a proper or infinitely near point.

We proceed by induction on the number of $\overline{\mathbf{k}}$-points blown up by $\eta$ and $\epsilon$, corresponding to the number of base points of $\varphi^{-1}$ and $\varphi$. If $\eta$ is an isomorphism, $\varphi$ is only a sequence of blow-ups in the boundary of $B$. Because of the nature of $B$ and $B^{\prime}$, this implies that $\varphi$ is either an isomorphism or a link of type I, from $X=\mathbb{P}^{2}$ to $X^{\prime}=\mathbb{F}_{1}$. Similarly, $\varphi$ is an isomorphism or a link of type III if $\epsilon$ is an isomorphism.

Thus, we may assume that $\eta$ (respectively, $\epsilon$ ) contracts at least one $(-1)$-curve of $Y$, not contracted by $\epsilon$ (respectively, $\eta$ ), which is thus the strict transform of an irreducible curve $E \subset B$ (respectively, $E^{\prime} \subset B^{\prime}$ ) of self-intersection at least -1 . If $E^{2}=-1$, we factor $\varphi$ through a link of type III from $X=\mathbb{F}_{1}$ to $\mathbb{P}^{2}$. We do the same with $\varphi^{-1}$ if $\left(E^{\prime}\right)^{2}=-1$.

Looking at the self-intersections of the curves on the boundaries $B$ and $B^{\prime}$, the remaining case is: only one (-1)-curve of $Y$ is contracted by $\eta$ (respectively, $\varepsilon$ ) and its image $E$ (respectively, $E^{\prime}$ ) under $\varepsilon$ (respectively, $\eta$ ) has self-intersection at least zero. This implies that $\varphi$ and $\varphi^{-1}$ have exactly one proper base point. If $E^{2}=1$, we factor $\varphi$ through a link of type I from $X=\mathbb{P}^{2}$ 


\section{JÉRÉMy BlanC AND IMMANUEl StAMPfli}

to $\mathbb{F}_{1}$. Otherwise, $E^{2}=0$, and we factor $\varphi$ through a link of type II from $X=\mathbb{F}_{n}$ to $\mathbb{F}_{n^{\prime}}$ with $n^{\prime}=n \pm 1$. It remains to see that $n^{\prime}=0$ is impossible. Indeed, otherwise the $(-1)$-curve of $X$ would not pass through the unique proper base point of $\varphi$ and would thus be sent by $\varphi$ onto a curve of self-intersection at least zero, not contracted by $\varphi^{-1}$.

Corollary 2.12. Let $\varphi:(X, B) \rightarrow\left(X^{\prime}, B^{\prime}\right)$ be a birational map between two natural completions of $\mathbb{A}^{2}$. All $\overline{\mathbf{k}}$-base points of $\varphi$ (that belong to $X$ as proper or infinitely near points) are defined over $\mathbf{k}$.

Proof. This follows from Proposition 2.10 and from the fact that the base points of any elementary link are defined over $\mathbf{k}$ (Lemma 2.9).

Let us recall how the result on elementary links gives the proof of Jung-van der Kulk's theorem (that we will not need in the remainder of this paper).

Corollary 2.13. The group Aut $\left(\mathbb{A}^{2}\right)$ is generated by the groups $\operatorname{Aff}\left(\mathbb{A}^{2}\right)$ and $\mathrm{J}=\bigcup_{n \geqslant 1} \mathrm{~J}_{n}$.

Proof. We view $g \in \operatorname{Aut}\left(\mathbb{A}^{2}\right)$ as a birational map $\left(\mathbb{P}^{2}, L_{\mathbb{P}^{2}}\right) \rightarrow\left(\mathbb{P}^{2}, L_{\mathbb{P}^{2}}\right)$. If it is an isomorphism of pairs, then $g \in \operatorname{Aff}\left(\mathbb{A}^{2}\right)$. Otherwise, we use Proposition 2.10 and write it as $g=\varphi_{m} \cdots \varphi_{1}$, where the $\varphi_{i}$ are elementary links. Denote by $r$ the smallest integer such that $\varphi_{r}$ is of type III. Then, $\varphi_{r-1} \cdots \varphi_{2}:\left(\mathbb{F}_{1}, E_{1} \cup L_{\mathbb{F}_{1}}\right) \rightarrow\left(\mathbb{F}_{1}, E_{1} \cup L_{\mathbb{F}_{1}}\right)$ is a composition of links of type II, which preserves the fibration $\mathbb{F}_{1} \rightarrow \mathbb{P}^{1}$, and the map $\varphi_{r} \cdots \varphi_{1}:\left(\mathbb{P}^{2}, L_{\mathbb{P}^{2}}\right)-\rightarrow\left(\mathbb{P}^{2}, L_{\mathbb{P}^{2}}\right)$ thus sends the lines through the point $q_{1} \in L_{\mathbb{P}^{2}}$ blown up by $\varphi_{1}$ onto the lines through the point $q_{2} \in L_{\mathbb{P}^{2}}$ blown up by $\left(\varphi_{r}\right)^{-1}$. Choosing $\alpha, \beta \in \operatorname{Aut}\left(\mathbb{P}^{2}, L_{\mathbb{P}^{2}}\right)$ that send $q_{1}, q_{2}$, respectively, onto $(0: 1: 0)$, the map $\beta \varphi_{r} \cdots \varphi_{1} \alpha^{-1}$ preserves the lines through $(0: 1: 0)$. Hence, its restriction to $\mathbb{A}^{2}$ belongs to J. Since the restriction of $\alpha, \beta$ belongs to $\operatorname{Aff}\left(\mathbb{A}^{2}\right)$, the map $\varphi_{r} \cdots \varphi_{1}$ belongs to the group generated by $\operatorname{Aff}\left(\mathbb{A}^{2}\right)$ and J. Proceeding by induction on $m$, we get the result.

For our purpose, we will need the decomposition into elementary links given by Proposition 2.10, but we will also need to have more precise information on the composition of links and their base points, provided by Lemma 2.14 and Proposition 2.16 below.

Lemma 2.14. Let $\varphi:\left(X_{0}, B_{0}\right) \rightarrow\left(X_{1}, B_{1}\right)$ and $\psi:\left(X_{1}, B_{1}\right) \rightarrow\left(X_{2}, B_{2}\right)$ be two elementary links. The birational map $\psi \circ \varphi$ is an isomorphism if and only if one of the following occurs:

(i) We have $X_{1}=\mathbb{F}_{1}$ and $X_{0}=X_{2}=\mathbb{P}^{2}$ (that is, $\psi$ and $\varphi^{-1}$ are both of type III).

(ii) The maps $\psi$ and $\varphi^{-1}$ are both of type I or both of type II, and share the same base point.

Moreover, if $X_{1}=\mathbb{F}_{n}$ and $X_{0}=X_{2}=\mathbb{F}_{n+1}$, for some $n \geqslant 1$, the map $\psi \circ \varphi$ is always an isomorphism.

Proof. If neither $\psi$ nor $\varphi^{-1}$ has a base point, we have $X_{1}=\mathbb{F}_{1}$ and $X_{0}=X_{2}=\mathbb{P}^{2}$, and both $\psi$ and $\varphi^{-1}$ are contractions of the $(-1)$-curve of $\mathbb{F}_{1}$. This implies that $\psi \circ \varphi$ is an automorphism of $\mathbb{P}^{2}$.

If $\psi$ has a base point which is not a base point of $\varphi^{-1}$, then $\psi \circ \varphi$ has a base point, and is thus not an isomorphism. The same holds after exchanging the roles of $\psi$ and $\varphi$.

The last case is when both $\psi$ and $\varphi^{-1}$ are links of type I or II and have the same base point. The description of the links (Lemma 2.9) implies that $\psi \circ \varphi$ is an isomorphism. It remains to see that this is always the case when $X_{1}=\mathbb{F}_{n}$ and $X_{0}=X_{2}=\mathbb{F}_{n+1}$. Indeed, this is true as the base point of every elementary link from $\left(\mathbb{F}_{n}, E_{n} \cup L_{\mathbb{F}_{n}}\right)$ to $\left(\mathbb{F}_{n+1}, E_{n+1} \cup L_{\mathbb{F}_{n+1}}\right)$ is the intersection point of $E_{n}$ and $L_{\mathbb{F}_{n}}$. 


\section{Automorphisms of the plane PRESERVing a CuRVE}

Definition 2.15. A decomposition of a birational map $\varphi:(X, B) \rightarrow\left(X^{\prime}, B^{\prime}\right)$ into elementary links is called reduced, if the composition of two consecutive elementary links is never an automorphism.

Proposition 2.16. Let $\varphi:(X, B) \rightarrow\left(X^{\prime}, B^{\prime}\right)$ be a birational map between two natural completions of $\mathbb{A}^{2}$, and let $\varphi=\varphi_{m} \cdots \varphi_{1}$ be a reduced decomposition of $\varphi$ into elementary links, with $m \geqslant 1$. Then, the following hold:

(i) The number $l$ of base points of $\varphi$ is equal to the number of elementary links $\varphi_{i}$ of type I or II. If $l \geqslant 1$, then $\varphi$ has a unique proper base point, which is equal to the base point of $\varphi_{1}$ if $\varphi_{1}$ is of type I or II, or to the preimage by $\varphi_{1}$ of the base point of $\varphi_{2}$ if $\varphi_{1}$ is of type III.

(ii) If $\varphi=\psi_{k} \cdots \psi_{1}$ is another decomposition into elementary links of $\varphi$, then $k \geqslant m$. Moreover, if $k=m$, the decomposition is unique, up to isomorphisms of completions.

Proof. We prove result (i) by induction on $m$, the case $m=1$ being obvious. Let $m \geqslant 2$.

Suppose that $\varphi_{1}$ is a link of type III. It contracts $E_{1} \subset X$ onto a point which is not a base point of $\varphi_{2}$, and thus not of $\varphi_{m} \cdots \varphi_{2}$, by the induction hypothesis. Consequently, the number of base points of $\varphi$ is equal to that of $\varphi_{m} \cdots \varphi_{2}$, and $\varphi$ has a unique proper base point. The latter is the preimage by $\varphi_{1}$ of the proper base point of $\varphi_{2}$.

Suppose now that $\varphi_{2}$ is a link of type III, which implies that $\varphi_{1}$ is a link $\mathbb{F}_{2} \rightarrow \mathbb{F}_{1}$ of type II, with one base point $q$. If $m=2$, the result is clear. If $m \geqslant 3$, we use the induction hypothesis and see that the unique proper base point of $\varphi_{m} \cdots \varphi_{2}$ is a point of $L_{\mathbb{F}_{1}} \backslash E_{1}$ which is not a base point of $\left(\varphi_{1}\right)^{-1}$. This implies that $\varphi$ has one more base point than $\varphi_{m} \cdots \varphi_{2}$. Moreover, since the proper base point of $\varphi_{m} \cdots \varphi_{2}$ is on $L_{\mathbb{F}_{1}}$ and since $L_{\mathbb{F}_{1}}$ is contracted by $\left(\varphi_{1}\right)^{-1}$ onto $q$, this implies that $q$ is the only proper base point of $\varphi$.

To prove result (i), it remains to study the case where $\varphi_{1}$ and $\varphi_{2}$ are links of type I or II. As in the previous case, $\varphi_{m} \cdots \varphi_{2}$ has a unique proper base point (here equal to that of $\varphi_{2}$ ), which

is not a base point of $\left(\varphi_{1}\right)^{-1}$ but belongs to the curve contracted by $\left(\varphi_{1}\right)^{-1}$ onto the base point of $\varphi_{1}$. This again implies that $\varphi$ has one more base point than $\varphi_{m} \cdots \varphi_{2}$ and that its unique proper base point is that of $\varphi_{1}$.

Now that result (i) has been proved, let us show that it implies result (ii). Suppose that another decomposition exists, that we can assume to be reduced, of length $k \leqslant m: \varphi=\varphi_{m} \cdots \varphi_{1}=$ $\psi_{k} \cdots \psi_{1}$. If $\varphi$ has no base point, both decompositions consist of one link of type III and the result is clear. Otherwise, the unique base point of $\varphi$ determines the first link, so we have $\psi_{1}=\alpha \varphi_{1}$ for some isomorphism of natural completions $\alpha$. Proceeding by induction, we get $k=m$ and the unicity of the decomposition.

Proposition 2.16 implies that the "length" of a reduced decomposition of $\varphi$ depends only on $\varphi$. The following definition is therefore natural.

Definition 2.17. The number of elementary links in a reduced decomposition of $\varphi$ is called the length of $\varphi$; we denote it by len $(\varphi)$.

\section{Birational maps preserving a curve and the proof of Theorem 1}

This section is devoted to the proof of Theorem 1, which is done using elementary links and by looking at the singularities of the curve obtained in the natural completions. 


\section{JÉRÉMy BLANC AND IMMANUEl StAMPfli}

Definition 3.1. If $\Gamma \subseteq X$ is a (closed) curve with no component in $B$ and $\varphi:(X, B)-\rightarrow(X, B)$ is a birational map, then we denote by $\varphi(\Gamma)$ the closure of $\varphi(\Gamma \cap X \backslash B)$ in $X$ and call it the image of $\Gamma$ under $\varphi$. Moreover, we denote by $\operatorname{Bir}((X, B), \Gamma)$ the group of birational maps $\varphi:(X, B)-\rightarrow$ $(X, B)$ such that $\varphi(\Gamma)=\Gamma$.

Remark 3.2. If $\Gamma \subset X$ is a curve having no component in $B$, then $\operatorname{Bir}((X, B), \Gamma)=$ $\{g \in \operatorname{Aut}(X \backslash B) \mid g(\Gamma \backslash B)=(\Gamma \backslash B)\}$. In particular, $\operatorname{Bir}((X, B), \Gamma)$ corresponds to the group $\operatorname{Aut}\left(\mathbb{A}^{2}, \Gamma \cap \mathbb{A}^{2}\right)$ of automorphisms of $X \backslash B=\mathbb{A}^{2}$ that preserve the closed curve $\Gamma \cap \mathbb{A}^{2}$ of $\mathbb{A}^{2}$.

To study maps preserving a curve, we will study the singularities of the curve on the boundary.

Definition 3.3. Let $\Gamma$ be a curve and let $p \in \Gamma$ be a $\overline{\mathbf{k}}$-point. We define the height $\operatorname{ht}_{\Gamma}(p)$ of $\Gamma$ at $p$ inductively, as follows:

(i) If $\Gamma$ is smooth at $p$, then we set $\operatorname{ht}_{\Gamma}(p):=0$.

(ii) Otherwise, let $\pi: \tilde{\Gamma} \rightarrow \Gamma$ be the blow-up of $\Gamma$ in $p$ and let $p_{1}, \ldots, p_{n}$ be the points of $\tilde{\Gamma}$ with $\pi\left(p_{i}\right)=p$. We set $\operatorname{ht}_{\Gamma}(p):=\max _{i}\left\{\operatorname{ht}_{\tilde{\Gamma}}\left(p_{i}\right)+1\right\}$.

Recall that for any curves $\Gamma_{1}, \Gamma_{2}$ having no common component on a smooth projective surface, the intersection number $\Gamma_{1} \cdot \Gamma_{2}$ is non-negative and corresponds to the sum of the intersection numbers $\left(\Gamma_{1} \cdot \Gamma_{2}\right)_{p}$, where $p$ runs over all $\overline{\mathbf{k}}$-points of $\Gamma_{1} \cap \Gamma_{2}$. The intersection number $\left(\Gamma_{1} \cdot \Gamma_{2}\right)_{p}$ satisfies $\left(\Gamma_{1} \cdot \Gamma_{2}\right)_{p} \geqslant m_{p}\left(\Gamma_{1}\right) \cdot m_{p}\left(\Gamma_{2}\right)$, where $m_{p}\left(\Gamma_{i}\right)$ is the multiplicity of $\Gamma_{i}$ at $p$, and equality holds if and only if the tangent cones of $\Gamma_{1}$ and $\Gamma_{2}$ at $p$ are distinct.

The next proposition is the key ingredient in the proof of Theorem 1.

Proposition 3.4. Let $\varphi:(X, B) \rightarrow\left(X^{\prime}, B^{\prime}\right)$ be a birational map between natural completions of $\mathbb{A}^{2}$, admitting a reduced decomposition $\varphi=\varphi_{m} \cdots \varphi_{1}$ into elementary links, with $m \geqslant 1$. Let $\Gamma \subset X$ be a curve having no component in $B$, and let $\Gamma^{\prime}=\varphi(\Gamma) \subset X^{\prime}$ be its image under $\varphi$. Suppose that one of the following holds:

(i) The link $\varphi_{1}$ is of type III (from $\mathbb{F}_{1}$ to $\mathbb{P}^{2}$ ) and $\left(E_{1} \cdot \Gamma\right)_{p_{0}}>1$, where $p_{0}$ is defined by $\left\{p_{0}\right\}=E_{1} \cap L_{\mathbb{F}_{1}}=: A$.

(ii) The link $\varphi_{1}$ is of type I or II, and the finite set $A \subset L_{X}$ of $\overline{\mathbf{k}}$-points lying on $\Gamma$ which are not (proper) base points of $\varphi$ satisfies $\sum_{p \in A}\left(L_{X} \cdot \Gamma\right)_{p}>1$.

Then, there exists a $\overline{\mathbf{k}}$-point $q \in L_{X^{\prime}}$ such that

$$
\operatorname{ht}_{\Gamma^{\prime}}(q) \geqslant l+\max _{p \in A} \mathrm{ht}_{\Gamma}(p),
$$

where $l$ is the number of base points of $\varphi^{-1}$. If $l \geqslant 1$, then $q$ can be chosen as the unique proper base point of $\varphi^{-1}$, which is a $\mathbf{k}$-point.

Proof. We proceed by induction on the number $m$ of elementary links in the reduced decomposition of $\varphi$. We distinguish the following cases, depending on the nature of the first link $\varphi_{1}$ :

(1) If $\varphi_{1}$ is a link of type III, it contracts the curve $E_{1} \subseteq \mathbb{F}_{1}$ onto a k-point $q \in \mathbb{P}^{2}$. By assumption, we have $\left(E_{1} \cdot \Gamma\right)_{p_{0}}>1$. The multiplicity of $\Gamma_{1}=\varphi_{1}(\Gamma)$ at $q$ is equal to $E_{1} \cdot \Gamma>1$, which implies that $\Gamma_{1}$ is singular at $q$ and that $\mathrm{ht}_{\Gamma_{1}}(q) \geqslant \mathrm{ht}_{\Gamma}\left(p_{0}\right)+1$.

If $m=1$, we have $l=1$ and $\operatorname{ht}_{\Gamma_{1}}(q) \geqslant \operatorname{ht}_{\Gamma}\left(p_{0}\right)+1=l+\max _{p \in A} \mathrm{ht}_{\Gamma}(p)$ (because $A=\left\{p_{0}\right\}$ ), so we are done. So, assume $m>1$. The point $q$ is not a base point of $\varphi_{2}$, since otherwise $\varphi_{2} \circ \varphi_{1}$ 


\section{Automorphisms of the Plane PRESERVING A CURVE}

would be an automorphism of $\mathbb{F}_{1}$. Moreover, $\left(L_{\mathbb{P}^{2}} \cdot \Gamma_{1}\right)_{q}>1$ because $\Gamma_{1}$ is singular at $q$. The claim follows by applying the induction hypothesis to $\varphi_{m} \cdots \varphi_{2}$, as $q$ is not a base point of $\varphi_{m} \cdots \varphi_{2}$.

(2) Suppose that $\varphi_{1}$ is a link of type II, that is, $\varphi_{1}: \mathbb{F}_{n^{\prime} \rightarrow-\rightarrow} \mathbb{F}_{n}$, where $n^{\prime}=n \pm 1$. By definition there exist blow-ups $\varepsilon: S \rightarrow \mathbb{F}_{n^{\prime}}$ and $\eta: S \rightarrow \mathbb{F}_{n}$ of k-points $q^{\prime} \in L_{\mathbb{F}_{n^{\prime}}}$ and $q \in L_{\mathbb{F}_{n}}$, respectively, such that $\varphi_{1}=\eta \circ \varepsilon^{-1}$. Denote by $\tilde{\Gamma} \subseteq S$ the strict transform of $\Gamma$ under $\varepsilon^{-1}$, and by $E_{q} \subset S$ the irreducible curve contracted by $\eta$ onto $q$, which is the strict transform of $L_{\mathbb{F}_{n^{\prime}}}$ under $\varepsilon^{-1}$. The map $\varepsilon^{-1}$ is an isomorphism in a neighbourhood of every point of $A \subset L_{\mathbb{F}_{n^{\prime}}}$. By hypothesis, one has $\sum_{p \in A}\left(L_{\mathbb{F}_{n^{\prime}}} \cdot \Gamma\right)_{p}>1$, which implies that $\tilde{\Gamma} \cdot E_{q}>1$ on $S$. The curve $\Gamma_{1}=\eta(\tilde{\Gamma}) \subset \mathbb{F}_{n}$ is thus singular at $q$, and the height of $\Gamma_{1}$ at $q$ is at least equal to $1+\max _{p \in A} h_{\Gamma}(p)$. If $m=1$, then we are done. If $m>1, q$ is not a base point of $\varphi_{2}$, since otherwise $\varphi_{2} \circ \varphi_{1}$ would be an isomorphism. Moreover, $q$ is a singular point of $\Gamma_{1}$ which belongs to $L_{\mathbb{F}_{n}}$, and if $\varphi_{2}$ is of type III, then $n=1$ and $n^{\prime}=2$, so $q$ is the intersection point of $E_{1}$ and $L_{\mathbb{F}_{1}}$. In any case, the point $q$ belongs to the set " $A$ " associated with $\varphi_{m} \cdots \varphi_{2}$, so the result follows by induction.

(3) The last case is when $\varphi_{1}$ is of type $I$, that is, when it is a map $\varphi_{1}: \mathbb{P}^{2} \rightarrow \mathbb{F}_{1}$. If $m=1$, then $l=0$ and the result is obvious, by choosing $q$ as the image of a point of $A$ with a maximal height. If $m>1$, the link $\varphi_{2}$ is a map $\mathbb{F}_{1} \rightarrow \mathbb{F}_{2}$ centred at the intersection point of $E_{1}$ and $L_{\mathbb{F}_{1}}$. In particular, the map $\varphi_{1}$ induces an isomorphism at a neighbourhood of any point of $A$, sending $L_{\mathbb{P}^{2}}$ onto $L_{\mathbb{F}_{1}}$, and sends the set $A$ onto the set " $A$ " associated with $\varphi_{m} \cdots \varphi_{2}$. The result follows by the induction hypothesis.

The following corollary is a direct consequence of Proposition 3.4.

Corollary 3.5. Let $\varphi \in \operatorname{Bir}((X, B), \Gamma) \backslash \operatorname{Aut}(X, B)$ and let $\varphi=\varphi_{m} \cdots \varphi_{1}$ be its reduced decomposition into elementary links. We assume that $\varphi_{1}$ is of type I or II, denote by $q$ the base point of $\varphi_{1}$ (which is the base point of $\varphi$ ) and denote by $A \subset L_{X}$ the finite set of $\overline{\mathbf{k}}$-points lying on $\Gamma$ which are not (proper) base points of $\varphi$. Then one of the following holds:

(i) The set $A$ is empty or it consists of one point $p$ which satisfies $\left(L_{X} \cdot \Gamma\right)_{p}=1$.

(ii) The set $A$ is non-empty and the height of $\Gamma$ at $q$ is greater than the height of $\Gamma$ at every point of $A$ (and thus $\Gamma$ is singular at $q$ ).

Proposition 3.6. Let $(X, B)$ be a natural completion of $\mathbb{A}^{2}$ and let $\Gamma$ be a curve in $X$ having no component in $B$. Then, there exist a natural completion $\left(X^{\prime}, B^{\prime}\right)$ of $\mathbb{A}^{2}$ and a birational map $\varphi:(X, B) \rightarrow\left(X^{\prime}, B^{\prime}\right)$ such that one of the following holds:

(i) $\operatorname{Bir}\left(\left(X^{\prime}, B^{\prime}\right), \varphi(\Gamma)\right) \subseteq \operatorname{Aut}\left(X^{\prime}, B^{\prime}\right)$.

(ii) The curve $\varphi(\Gamma)$ intersects transversally $L_{X^{\prime}}$, that is, $\left(\varphi(\Gamma) \cdot L_{X^{\prime}}\right)_{p} \leqslant 1$ for any $p \in X^{\prime}(\overline{\mathbf{k}})$.

Proof. Let $\left(X_{0}, B_{0}\right)=(X, B)$ and let $\varphi_{0}=\mathrm{id} \in \operatorname{Aut}\left(X_{0}, B_{0}\right)$. We construct inductively a sequence of elementary links

$$
\left(X_{0}, B_{0}\right) \stackrel{\varphi_{1}}{-\rightarrow}\left(X_{1}, B_{1}\right)_{-\rightarrow}^{\varphi_{2}} \rightarrow\left(X_{2}, B_{2}\right)_{-\rightarrow}^{\varphi_{3}} \rightarrow \ldots
$$

Let $i \geqslant 0$ and assume that $\varphi_{0}, \ldots, \varphi_{i}$ have already been constructed. We write $\Gamma_{i}=\varphi_{i} \cdots \varphi_{1}(\Gamma)$, $G_{i}=\left(\operatorname{Bir}\left(X_{i}, B_{i}\right), \Gamma_{i}\right)$ and define $\varphi_{i+1}$ in the following way:

(1) If $G_{i} \subseteq \operatorname{Aut}\left(X_{i}, B_{i}\right)$ or $\Gamma_{i}$ intersects transversally $L_{X_{i}}$, we set $\left(X_{i+1}, B_{i+1}\right)=\left(X_{i}, B_{i}\right)$ and $\varphi_{i+1}=\mathrm{id}$.

(2) If case (1) does not hold and the reduced decomposition into elementary links of every $g \in G_{i} \backslash \operatorname{Aut}\left(X_{i}, B_{i}\right)$ starts with an elementary link $\tau_{0}: \mathbb{F}_{1} \rightarrow \mathbb{P}^{2}$ of type III, then we set $\varphi_{i+1}=\tau_{0}$, and thus set $\left(X_{i+1}, B_{i+1}\right)=\left(\mathbb{P}^{2}, L_{\mathbb{P}^{2}}\right)$. 


\section{JÉRÉMy BLANC AND IMMANUEl StAMPfli}

(3) If cases (1) and (2) do not occur, there exists a $g \in G_{i} \backslash \operatorname{Aut}\left(X_{i}, B_{i}\right)$ such that the reduced decomposition into elementary links of $g$ starts with an elementary link $\tau_{1}$, not of type III. In this case we set $\varphi_{i+1}=\tau_{1}$, and let $\left(X_{i+1}, B_{i+1}\right)$ be the target of $\tau_{1}$.

Now, we claim that the sequence $(*)$ satisfies the following two properties:

(a) If $\varphi_{i+1}$ is not an automorphism, then we have for all $g \in G_{i}$,

$$
\operatorname{len}\left(\varphi_{i+1} g \varphi_{i+1}^{-1}\right)<\operatorname{len}(g) \text { or } \operatorname{len}\left(\varphi_{i+1} g \varphi_{i+1}^{-1}\right)=\operatorname{len}(g)=0 .
$$

(b) The sequence $(*)$ is stationary after finitely many steps; that is, $\varphi_{i}$ is an automorphism for $i$ large enough.

Property (a) will serve to show property (b), which directly implies the result. It thus remains to prove these two properties.

Proof of property (a). In case (1), there is nothing to prove.

If we are in case (2), then for all $g \in G_{i} \backslash \operatorname{Aut}\left(X_{i}, B_{i}\right)$, the reduced decomposition into elementary links starts with $\tau_{0}: \mathbb{F}_{1} \rightarrow \mathbb{P}^{2}$ and ends with $\left(\tau_{0}\right)^{-1}$, since $G_{i}$ is a group. The conjugation of $G_{i}$ by $\tau_{0}$ decreases the length of any element of $G_{i} \backslash \operatorname{Aut}\left(X_{i}, B_{i}\right)$ by two. Moreover, $\left(X_{i}, B_{i}\right)=\left(\mathbb{F}_{1}, E_{1} \cup L_{\mathbb{F}_{1}}\right)$, so $\operatorname{Aut}\left(X_{i}, B_{i}\right)$ preserves the curve $E_{1}$ contracted by $\tau_{0}$, which implies that $\tau_{0} \operatorname{Aut}\left(X_{i}, B_{i}\right)\left(\tau_{0}\right)^{-1} \subset \operatorname{Aut}\left(\mathbb{P}^{2}, L_{\mathbb{P}^{2}}\right)=\operatorname{Aut}\left(X_{i+1}, B_{i+1}\right)$. It follows that property (a) is satisfied.

Assume that we are in case (3). As we are not in case (1), there exists a $p_{0} \in X_{i}$ such that $\left(\Gamma_{i} \cdot L_{X_{i}}\right)_{p_{0}}>1$. Moreover, by definition of case (3), there exists a $g \in G_{i} \backslash \operatorname{Aut}\left(X_{i}, B_{i}\right)$ such that the reduced decomposition into elementary links starts with $\varphi_{i+1}:\left(X_{i}, B_{i}\right) \rightarrow\left(X_{i+1}, B_{i+1}\right)$, and $\varphi_{i+1}$ has a base point $p$ since it is not of type III. Applying Corollary 3.5 to $g$, we see that $\left(\Gamma_{i} \cdot L_{X_{i}}\right)_{p}>1$, and that either the height of $\Gamma_{i}$ at $p$ is greater than the height of $\Gamma_{i}$ at every other point of $\Gamma_{i} \cap L_{X_{i}}$ and $\Gamma_{i}$ is singular at $p$, or all points in $\Gamma_{i} \cap L_{X_{i}} \backslash\{p\}$ intersect transversally $L_{X_{i}}$. This implies that any element $h \in \operatorname{Aut}\left(X_{i}, B_{i}\right) \cap G_{i}$ fixes $p$, and thus satisfies $\varphi_{i+1} h \varphi_{i+1}^{-1} \in$ $\operatorname{Aut}\left(X_{i+1}, B_{i+1}\right)$. We can then apply Corollary 3.5 to any element $h \in G_{i} \backslash \operatorname{Aut}\left(X_{i}, B_{i}\right)$ that does not start with a link of type III, and see that $p$ is the unique proper base point of $h$, so the reduced decomposition of $h$ starts with $\varphi_{i+1}$. It remains to observe that no element $h \in G_{i} \backslash \operatorname{Aut}\left(X_{i}, B_{i}\right)$ starts with a link $\tau$ of type III. Indeed, otherwise we would have $X_{i}=\mathbb{F}_{1}$ and $\varphi_{i+1}$ would be an elementary link from $\mathbb{F}_{1}$ to $\mathbb{F}_{2}$, so $p$ would be the intersection point $E_{1} \cap L_{\mathbb{F}_{1}}$. Since $\left(\Gamma_{i} \cdot L_{\mathbb{F}_{1}}\right)_{p}>1$, it would follow that $\left(\tau\left(\Gamma_{i}\right) \cdot L_{\mathbb{P}^{2}}\right)_{\tau(p)}>\left(\Gamma_{i} \cdot L_{\mathbb{F}_{1}}\right)_{p}>1$. We could then apply Proposition 3.4 to $h \tau^{-1}:\left(\mathbb{P}^{2}, L_{\mathbb{P}^{2}}\right) \rightarrow\left(\mathbb{F}_{1}, E_{1} \cup L_{\mathbb{F}_{1}}\right)$, and find some other point $p^{\prime} \in L_{\mathbb{F}_{1}}$ where $\Gamma_{i}$ would have height greater than $p$, or such that $\Gamma_{i}$ would be smooth at $p$ and $p^{\prime}$ and $\left(\Gamma_{i} \cdot L_{\mathbb{F}_{1}}\right)_{p^{\prime}}>\left(\Gamma_{i} \cdot L_{\mathbb{F}_{1}}\right)_{p}$, giving a contradiction. This yields property (a).

Proof of property (b)

Property (a) implies that for each $i$ such that $\varphi_{1}, \ldots, \varphi_{i}$ are not isomorphisms, the product $\varphi_{i} \cdots \varphi_{1}$ is a reduced decomposition into elementary links. For any $i \geqslant 0$ such that neither $\varphi_{i+1}$ nor $\varphi_{i+2}$ is an isomorphism, we define $p_{i} \in L_{X_{i}} \subset B_{i} \subset X_{i}$ as the unique proper base point of $\varphi_{i+2} \circ \varphi_{i+1}$.

Under the assumption that none of the three maps $\varphi_{i+1}, \varphi_{i+2}$ and $\varphi_{i+3}$ is an isomorphism, we show that $\left(\Gamma_{i} \cdot L_{X_{i}}\right)_{p_{i}} \geqslant\left(\Gamma_{i+1} \cdot L_{X_{i+1}}\right)_{p_{i+1}}$ and give some consequences when equality holds.

- The link $\varphi_{i+1}$ is of type II: We can write $\varphi_{i+1}=\eta \circ \pi^{-1}$, where $\pi: S \rightarrow \underset{\widetilde{N}_{i}}{X_{i}}$ is the blow-up of $p_{i}$ and $\eta: S \rightarrow X_{i+1}$ is the blow-up of a point $q \neq p_{i+1}$. Denote by $\widetilde{\Gamma_{i}}, \widetilde{L_{X_{i}}} \subset S$ the strict transforms of $\Gamma_{i}$ and $L_{X_{i}}$, and let $E_{p_{i}}$ be the exceptional divisor of $\pi$. It follows that $\eta$ 


\section{Automorphisms of the plane PRESERVing a CuRVE}

contracts $\widetilde{L_{X_{i}}}$. Note that $\eta^{-1}$ restricts to an isomorphism in a neighbourhood of $p_{i+1}$, which sends $L_{X_{i+1}}$ onto $E_{p_{i}}$. This yields the following estimate:

$$
\begin{aligned}
\left(\Gamma_{i} \cdot L_{X_{i}}\right)_{p_{i}} & \geqslant m_{p_{i}}\left(\Gamma_{i}\right)=\widetilde{\Gamma}_{i} \cdot E_{p_{i}} \\
& \geqslant\left(\widetilde{\Gamma_{i}} \cdot E_{p_{i}}\right)_{\eta^{-1}\left(p_{i+1}\right)}=\left(\Gamma_{i+1} \cdot L_{X_{i+1}}\right)_{p_{i+1}},
\end{aligned}
$$

where $m_{p_{i}}\left(\Gamma_{i}\right)$ denotes the multiplicity of $\Gamma_{i}$ in $p_{i}$. Moreover, if $\left(\Gamma_{i} \cdot L_{X_{i}}\right)_{p_{i}}=m_{p_{i}}\left(\Gamma_{i}\right)=$ $\left(\Gamma_{i+1} \cdot L_{X_{i+1}}\right)_{p_{i+1}}>1$, then $\mathrm{ht}_{\Gamma_{i}}\left(p_{i}\right)>\mathrm{ht}_{\Gamma_{i+1}}\left(p_{i+1}\right)$.

- The link $\varphi_{i+1}$ is of type I: Note that $\left(\varphi_{i+1}\right)^{-1}: \mathbb{F}_{1} \rightarrow \mathbb{P}^{2}$ is the blow-up of $p_{i} \in \mathbb{P}^{2}$ and that $\Gamma_{i+1}$ and $L_{\mathbb{F}_{1}}$ are the strict transforms of $\Gamma_{i}$ and $L_{\mathbb{P}^{2}}$ under $\left(\varphi_{i+1}\right)^{-1}$, respectively. It follows that $\left(\Gamma_{i} \cdot L_{\mathbb{P}^{2}}\right)_{p_{i}} \geqslant\left(\Gamma_{i+1} \cdot L_{\mathbb{F}_{1}}\right)_{p_{i+1}}$. Moreover, equality implies $\left(\Gamma_{i} \cdot L_{\mathbb{P}^{2}}\right)_{p_{i}}=$ $\left(\Gamma_{i+1} \cdot L_{\mathbb{F}_{1}}\right)_{p_{i+1}}=0$.

- The link $\varphi_{i+1}$ is of type III: Since $\varphi_{i+1}$ is an isomorphism in a neighbourhood of $p_{i}$ and $p_{i+1}=\varphi_{i+1}\left(p_{i}\right)$, it follows that $\left(\Gamma_{i} \cdot L_{\mathbb{F}_{1}}\right)_{p_{i}}=\left(\Gamma_{i+1} \cdot L_{\mathbb{P}^{2}}\right)_{p_{i+1}}$ and $\operatorname{ht}_{\Gamma_{i}}\left(p_{i}\right)=\operatorname{ht}_{\Gamma_{i+1}}\left(p_{i+1}\right)$.

Now, assume towards a contradiction, that the sequence $(*)$ is never stationary; that is, $\varphi_{i}$ is not an isomorphism for all $i \geqslant 1$. According to this case-by-case analysis, we see that $\left(\Gamma_{i} \cdot L_{X_{i}}\right)_{p_{i}}$ is a decreasing sequence in $i$ and for every $r>1$ there are only finitely many $i$ with $\left(\Gamma_{i} \cdot L_{X_{i}}\right)_{p_{i}}=r$. Thus, there exists an $I$ such that for all $i \geqslant I$ we have $\left(\Gamma_{i} \cdot L_{X_{i}}\right)_{p_{i}} \leqslant 1$. As the sequence $(*)$ is not stationary, we have a $\varphi_{i+1}$ with $i \geqslant I$ which is a link of type I or II. The point $p_{i}$ is thus the base point of $\varphi_{i+1}$ and satisfies $\left(\Gamma_{i} \cdot L_{X_{i}}\right)_{p_{i}} \leqslant 1$. By Corollary 3.5 applied to an element of $G_{i} \backslash \operatorname{Aut}\left(X_{i}, B_{i}\right)$ that starts with $\varphi_{i+1}$, this implies that $\left(\Gamma_{i} \cdot L_{X_{i}}\right)_{q}=1$ for all $q \in L_{X_{i}} \cap \Gamma_{i}$. Hence, we are in case (1), giving a contradiction.

LEMma 3.7. Let $(X, B)=\left(\mathbb{P}^{2}, L_{\mathbb{P}^{2}}\right)$, and let $\Gamma$ be a conic (as always, reduced but not necessarily irreducible) in $X=\mathbb{P}^{2}$ intersecting $L_{\mathbb{P}^{2}}$ in two distinct points (not necessarily defined over $\mathbf{k}$ ). Then, $\operatorname{Bir}((X, B), \Gamma) \subseteq \operatorname{Aut}(X, B)$.

Proof. Suppose, to the contrary, that there exists a $g \in \operatorname{Bir}((X, B), \Gamma)$ which is not an automorphism of $\mathbb{P}^{2}$.

Applying Proposition 3.4, one of the two points of $\Gamma \cap L_{X}$ is a base point of $g$ (and, in particular, is defined over k). By Proposition 2.10 and Lemma 2.14, there exists an integer $n \geqslant 2$ such that the reduced decomposition of $g$ into elementary links starts with $\varphi_{2 n} \varphi_{2 n-1} \cdots \varphi_{1}$, where $\varphi_{1}: \mathbb{P}^{2} \rightarrow \mathbb{F}_{1}, \varphi_{2 n}: \mathbb{F}_{1} \rightarrow \mathbb{P}^{2}, \varphi_{i}: \mathbb{F}_{i-1} \rightarrow \mathbb{F}_{i}$ and $\varphi_{i+n-1}: \mathbb{F}_{n-i+2} \rightarrow \mathbb{F}_{n-i+1}$ for $i=2, \ldots, n$. The curve $\varphi_{1}(\Gamma)$ intersects transversally $E_{1}$ in one point and $L_{\mathbb{F}_{1}}$ in one point, and does not pass through the intersection point of $E_{1}$ and $L_{\mathbb{F}_{1}}$, blown up by $\varphi_{2}$. This implies that the same holds for $\varphi_{2} \varphi_{1}(\Gamma)$, with $E_{2}$ and $L_{\mathbb{F}_{2}}$, and that $\varphi_{2} \varphi_{1}(\Gamma)$ passes through the point blown up by $\left(\varphi_{2}\right)^{-1}$. Proceeding by induction, the curve $\varphi_{n} \cdots \varphi_{1}(\Gamma) \subset \mathbb{F}_{n}$ intersects transversally $E_{n}$ in one point and $L_{\mathbb{F}_{n}}$ in one point, and passes through the point blown-up by $\left(\varphi_{n}\right)^{-1}$. Consequently, it does not pass through the point blown-up by $\varphi_{n+1}$, which implies that $\varphi_{n+1} \varphi_{n} \cdots \varphi_{1}(\Gamma) \subset \mathbb{F}_{n-1}$ intersects $E_{n-1} \cup L_{\mathbb{F}_{n-1}}$ in two distinct points, both on $E_{n-1}$. Proceeding by induction, the curve $\varphi_{2 n-1} \cdots \varphi_{n} \cdots \varphi_{1}(\Gamma) \subset \mathbb{F}_{1}$ intersects $E_{1} \cup L_{\mathbb{F}_{1}}$ in two distinct points, both on $E_{1}$. The curve $\varphi_{2 n} \cdots \varphi_{1}(\Gamma) \subset \mathbb{P}^{2}$ intersects $L_{\mathbb{P}^{2}}$ in one point $q$, and is singular at this point, with two branches. The remaining part of the decomposition of $g$ is equal to $\varphi_{m} \cdots \varphi_{2 n+1}$, and its unique proper base point is different from $q$. Proposition 3.4 implies that $g(\Gamma)$ is singular at a point of $B=L_{\mathbb{P}^{2}}$, which gives a contradiction.

Proposition 3.8. Let $(X, B)$ be a natural completion of $\mathbb{A}^{2}$, and let $\Gamma$ be a curve in $X$. If $\Gamma$ intersects transversally $L_{X}$, then there exist a natural completion $\left(X^{\prime}, B^{\prime}\right)$ of $\mathbb{A}^{2}$ and a birational map $\varphi:(X, B) \rightarrow\left(X^{\prime}, B^{\prime}\right)$ such that one of the following holds: 


\section{JÉRÉMy BlanC AND IMMANUEl StAMPfli}

(i) $\operatorname{Bir}\left(\left(X^{\prime}, B^{\prime}\right), \varphi(\Gamma)\right) \subseteq \operatorname{Aut}\left(X^{\prime}, B^{\prime}\right)$.

(ii) We have $X^{\prime}=\mathbb{P}^{2}$ and $\varphi(\Gamma) \subset \mathbb{P}^{2}$ is defined by a polynomial in $\mathbf{k}[x]$.

Proof. We can assume the existence of a $g \in \operatorname{Bir}((X, B), \Gamma) \backslash \operatorname{Aut}(X, B)$ admitting a reduced decomposition $g=\varphi_{m} \cdots \varphi_{1}$ into elementary links.

(1) Suppose $X=\mathbb{P}^{2}$. By Corollary 3.5, the curve $\Gamma$ intersects $L_{X}$ in at most two points, hence $\Gamma$ is a line or a conic. If $\Gamma$ is a line, the equation can be chosen to be $x=0$, and if $\Gamma$ is a conic, the result follows from Lemma 3.7.

(2) Suppose $X \not \mathbb{P}^{2}$ but that there exists an $1 \leqslant i \leqslant m$ such that $\varphi_{i}$ is a link $\varphi_{i}: \mathbb{F}_{1} \rightarrow \mathbb{P}^{2}$ of type III, contracting $E_{1}$ onto a point $q \in L_{\mathbb{P}^{2}}$. If $\Gamma^{\prime}=\varphi_{i} \cdots \varphi_{1}(\Gamma)$ intersects transversally $L_{\mathbb{P}^{2}}$, we conclude by applying case (1) to $\Gamma^{\prime}$. There thus exists a point $p \in L_{\mathbb{P}^{2}}$ with $\left(\Gamma^{\prime} \cdot L_{\mathbb{P}^{2}}\right)_{p}>1$. We claim that $p$ can be chosen to be equal to $q$. If $i=1$, this is true because $\Gamma^{\prime}=\varphi_{1}(\Gamma)$ and $\Gamma$ intersects transversally $L_{\mathbb{F}_{1}}$, which is the strict transform of $L_{\mathbb{P}^{2}}$. If $i>1$, the claim follows from Proposition 3.4, applied to $\left(\varphi_{1}\right)^{-1} \cdots\left(\varphi_{i}\right)^{-1}:\left(\mathbb{P}^{2}, L_{\mathbb{P}^{2}}\right)-\rightarrow(X, B)$. Since $\varphi_{i+1}$ is a link of type I and $q$ is not a base point of $\varphi_{i+1}, \varphi_{i+1}\left(\Gamma^{\prime}\right)$ does not intersect transversally $L_{\mathbb{F}_{1}}$. This implies that $m \geqslant i+2$, and that $\left(\varphi_{m} \cdots \varphi_{i+1}\right)^{-1}$ has at least one base point. Applying Proposition 3.4 to $\varphi_{m} \cdots \varphi_{i+1}$, we see that the curve $\Gamma=\varphi_{m} \cdots \varphi_{i+1}\left(\Gamma^{\prime}\right)$ has a singular point at the proper base point of $\left(\varphi_{m} \cdots \varphi_{i+1}\right)^{-1}$. Since this lies on $L_{X}$, we get a contradiction.

(3) We can now assume $X=\mathbb{F}_{n}$ for some $n \geqslant 1$ and that all $\varphi_{i}$ are links of type II. By Corollary 3.5, $L_{\mathbb{F}_{n}} \cap \Gamma$ contains at most two points and if it contains two, then one of these is a base point of $\varphi_{1}$. If one of the points of $L_{\mathbb{F}_{n}} \cap \Gamma$ is the intersection point $p_{n}$ of $L_{\mathbb{F}_{n}}$ and $E_{n}$, we perform an elementary link $\psi: \mathbb{F}_{n} \rightarrow \mathbb{F}_{n+1}$. Because $L_{\mathbb{F}_{n}} \cap \Gamma$ contains at most two points, the curve $\psi(\Gamma)$ intersects transversally $L_{\mathbb{F}_{n+1}}$, in at most two points. Moreover, writing $\left\{p_{n+1}\right\}=L_{\mathbb{F}_{n+1}} \cap E_{n+1}$, we obtain $\left(\psi(\Gamma) \cdot E_{n+1}\right)_{p_{n+1}}=\left(\Gamma \cdot E_{n}\right)_{p_{n}}-1$. Performing a sequence of elementary links if needed, we reduce to the case where $L_{\mathbb{F}_{n}} \cap \Gamma$ contains at most two points, none of which belongs to $E_{n}$.

If $L_{\mathbb{F}_{n}} \cap \Gamma$ is empty, then $\Gamma$ is contained in a finite set of fibres of $\mathbb{F}_{n} \rightarrow \mathbb{P}^{1}$. Going from $\mathbb{F}_{n}$ to $\mathbb{F}_{1}$ and then to $\mathbb{P}^{2}$, we send the fibres onto lines of the form $x=a$ where $a \in \overline{\mathbf{k}}$, and see that $L_{\mathbb{F}_{n}}$ has equation in $\mathbf{k}[x]$.

It remains to see that it is not possible, in the case where $L_{\mathbb{F}_{n}} \cap \Gamma$ contains one or two points that belong to $L_{\mathbb{F}_{n}} \backslash E_{n}$, to have a $g \in \operatorname{Bir}((X, B), \Gamma) \backslash \operatorname{Aut}(X, B)$ whose reduced decomposition consists of only links of type II. Such an element has a decomposition $g=\varphi_{2 k} \cdots \varphi_{1}$, where $k \geqslant 1, \varphi_{i}$ is a link $\mathbb{F}_{n+i-1} \rightarrow \mathbb{F}_{n+i}$ for $i=1, \ldots, k, \varphi_{i}$ is a link $\mathbb{F}_{n+2 k-i+1} \rightarrow \mathbb{F}_{n+2 k-i}$ for $i=k+1, \ldots, 2 k$ and where $\left(\varphi_{k}\right)^{-1}$ and $\varphi_{k+1}$ do not have the same base point (see Lemma 2.14). In particular, the base point of $\varphi_{1}$ is the intersection point of $E_{n}$ and $L_{\mathbb{F}_{n}}$, and does not lie on $\Gamma$, so $\varphi_{1}(\Gamma)$ passes through the base point of $\left(\varphi_{1}\right)^{-1}$, which is not a base point of $\varphi_{2}$. By induction, we deduce that $\left(\varphi_{i} \cdots \varphi_{1}\right)(\Gamma)$ passes through the base point of $\left(\varphi_{i}\right)^{-1}$, for $i=1, \ldots, 2 k$. In the case $i=2 k$, this implies that $g(\Gamma)$ passes through the base point of $\left(\varphi_{2 k}\right)^{-1}$, that is, through the intersection point of $E_{n}$ and $L_{\mathbb{F}_{n}}$, contradicting the fact that $g(\Gamma)=\Gamma$.

Corollary 3.9. Let $(X, B)$ be a natural completion of $\mathbb{A}^{2}$ and let $\Gamma$ be a curve in $X$ having no component in $B$. Then, there exist a natural completion $\left(X^{\prime}, B^{\prime}\right)$ of $\mathbb{A}^{2}$ and a birational map $\varphi:(X, B) \rightarrow\left(X^{\prime}, B^{\prime}\right)$ such that one of the following holds:

(i) $\operatorname{Bir}\left(\left(X^{\prime}, B^{\prime}\right), \varphi(\Gamma)\right) \subseteq \operatorname{Aut}\left(X^{\prime}, B^{\prime}\right)$.

(ii) We have $X^{\prime}=\mathbb{P}^{2}$ and $\varphi(\Gamma) \subset \mathbb{P}^{2}$ is defined by a polynomial in $\mathbf{k}[x]$.

Proof. This follows directly from Propositions 3.6 and 3.8. 


\section{Automorphisms of the Plane PRESERVing A CURVE}

Theorem 1 is now a direct consequence of Corollary 3.9.

Proof of Theorem 1. In the case where the equation of $\Gamma$ is in $\mathbf{k}[x]$, that is, when $\Gamma$ is a fence, the explicit description of $\operatorname{Aut}\left(\mathbb{A}^{2}, \Gamma\right)$ is an easy calculation. If $\Gamma$ is not equivalent to a fence by an automorphism of $\mathbb{A}^{2}$, Corollary 3.9 and Lemma 2.6 imply that we can conjugate $\operatorname{Aut}\left(\mathbb{A}^{2}, \Gamma\right)$ to a subgroup of $\operatorname{Aff}\left(\mathbb{A}^{2}\right)$ or $J_{n}$ for some $n$. This implies that $\operatorname{Aut}\left(\mathbb{A}^{2}, \Gamma\right)$ is an algebraic group. Moreover, we obtain a morphism of algebraic groups $\operatorname{Aut}\left(\mathbb{A}^{2}, \Gamma\right) \rightarrow \operatorname{Aut}(\Gamma)$. It remains to observe that curves fixed pointwise by elements of $J_{n}$ and $\operatorname{Aff}\left(\mathbb{A}^{2}\right)$ are fences in a suitable coordinate system of $\mathbb{A}^{2}$.

\subsection{Generalisation to other subsets}

Definition 3.10. If $\Delta \subset \mathbb{A}^{2}(\overline{\mathbf{k}})$ is any subset, we denote by $\operatorname{Aut}\left(\mathbb{A}^{2}, \Delta\right)$ the group of automorphisms of $\mathbb{A}^{2}$ that leave the set $\Delta$ invariant, and by $\operatorname{Aut}_{F}\left(\mathbb{A}^{2}, \Delta\right)$ the group of automorphisms of $\mathbb{A}^{2}$ fixing any element of $\Delta$.

By definition, $\operatorname{Aut}_{F}\left(\mathbb{A}^{2}, \Delta\right)$ is always a normal subgroup of $\operatorname{Aut}\left(\mathbb{A}^{2}, \Delta\right)$. If $\operatorname{Aut}\left(\mathbb{A}^{2}, \Delta\right)$ is an algebraic group, then $\operatorname{Aut}_{F}\left(\mathbb{A}^{2}, \Delta\right)$ is moreover an algebraic subgroup. Theorem 1 implies the following result.

Proposition 3.11. Let $\Delta \subset \mathbb{A}^{2}(\overline{\mathbf{k}})$ be a subset. Applying an element $\varphi \in \operatorname{Aut}\left(\mathbb{A}^{2}\right)$, one of the following holds:

(i) The set $\Delta$ is contained in a fence given by $f(x)=0$, where $f \in \mathbf{k}[x]$, and the group $\operatorname{Aut}_{F}\left(\mathbb{A}^{2}, \Delta\right)$ is not algebraic; it contains the group

$$
\{(x, y) \mapsto(x, y+p(x) f(x)) \mid p \in \mathbf{k}[x]\} \simeq \mathbf{k}[x] .
$$

(ii) The group $\operatorname{Aut}\left(\mathbb{A}^{2}, \Delta\right)$ is equal to $\left\{g \in \operatorname{Aff}\left(\mathbb{A}^{2}\right) \mid g(\Delta)=\Delta\right\}$ and is thus an algebraic subgroup of $\operatorname{Aff}\left(\mathbb{A}^{2}\right)$. Moreover, $\operatorname{Aut}_{F}\left(\mathbb{A}^{2}, \Delta\right)$ is trivial.

(iii) The group $\operatorname{Aut}\left(\mathbb{A}^{2}, \Delta\right)$ is equal to $\left\{g \in \mathrm{J}_{n} \mid g(\Delta)=\Delta\right\}$ for an integer $n \geqslant 1$ and is thus an algebraic subgroup of $\mathrm{J}_{n}$. Moreover, $\operatorname{Aut}_{F}\left(\mathbb{A}^{2}, \Delta\right)$ is trivial.

Proof. Denote by $I(\Delta) \subset \mathbf{k}[x, y]$ the ideal of polynomials vanishing on $\Delta$, and by $\bar{\Delta} \subset \mathbb{A}^{2}(\overline{\mathbf{k}})$ the closure of $\Delta$, which is the set of points where $I(\Delta)$ vanishes. Then we obtain $\operatorname{Aut}\left(\mathbb{A}^{2}, \Delta\right)=$ $\operatorname{Aut}\left(\mathbb{A}^{2}, \bar{\Delta}\right)$ and $\operatorname{Aut}_{F}\left(\mathbb{A}^{2}, \Delta\right)=\operatorname{Aut}_{F}\left(\mathbb{A}^{2}, \bar{\Delta}\right)$. We can thus replace $\Delta$ with $\bar{\Delta}$.

If $\Delta$ is a finite union of points, we get case (i). Otherwise, $\Delta$ consists of one curve $\Gamma$ (reduced but not necessarily irreducible) and a finite number of points of $\mathbb{A}^{2}(\overline{\mathbf{k}})$. Thus, $\operatorname{Aut}\left(\mathbb{A}^{2}, \Delta\right) \subset$ $\operatorname{Aut}\left(\mathbb{A}^{2}, \Gamma\right)$. The result then follows from the description of $\Gamma$ and $\operatorname{Aut}\left(\mathbb{A}^{2}, \Gamma\right)$, given in Theorem 1.

In the case where $\Delta$ is finite, the group $\operatorname{Aut}\left(\mathbb{A}^{2}, \Delta\right)$ is quite big; indeed, it is often maximal. This is the case for example when $\mathbf{k}=\mathbb{C}$, as pointed out to us by J.-P. Furter and P.-M. Poloni. This is a consequence of the following observation.

LEMma 3.12. Let $G$ be a group acting on a set $S$. Let $\Delta \subset S$ be a finite subset of $r \geqslant 1$ points. Suppose that $G$ acts $2 r$-transitively on $S$ and that $|S|>2 r$. Then,

$$
G_{\Delta}=\{g \in G \mid g(\Delta)=\Delta\}
$$

is a maximal subgroup of $G$. 


\section{JÉRÉMy BLANC AND IMMANUEl StAMPfli}

Proof. Since $G_{\Delta}$ is not trivial and not equal to $G$ (because of the $r$-transitivity), it suffices to take an $a \in G \backslash G_{\Delta}$ and show that $a$ and $G_{\Delta}$ generate $G$. We can write $\Delta=\left\{x_{1}, \ldots, x_{r}\right\}$, with $a\left(x_{1}\right), \ldots, a\left(x_{k}\right) \notin \Delta$ and $a\left(x_{k+1}\right), \ldots, a\left(x_{r}\right) \in \Delta$, where $1 \leqslant k \leqslant r$. The hypotheses yield the existence of a $g \in G$ that fixes $x_{1}, \ldots, x_{r}, a\left(x_{2}\right), \ldots, a\left(x_{k}\right)$ and does not fix $a\left(x_{1}\right)$. Then, $g \in G_{\Delta}$ and $f=a^{-1} g a$ fixes $x_{2}, \ldots, x_{r}$ but $f\left(x_{1}\right) \notin \Delta$.

It remains to see that any $h \in G \backslash G_{\Delta}$ is generated by $f$ and $G_{\Delta}$. We write $\Delta=\left\{z_{1}, \ldots, z_{r}\right\}$, with $h\left(z_{1}\right), \ldots, h\left(z_{j}\right) \notin \Delta$ and $h\left(z_{j+1}\right), \ldots, h\left(z_{r}\right) \in \Delta$, where $1 \leqslant j \leqslant r$. Replacing $h$ with its composition with an element of $G_{\Delta}$, we can assume that $h\left(z_{i}\right)=z_{i}$ for $i=j+1, \ldots, r$. For $i=1, \ldots, j$, we choose a $g_{i} \in G_{\Delta}$ that sends $z_{i}$ onto $x_{1}$ and sends $h\left(z_{i}\right)$ onto $f\left(x_{1}\right)$. Then, $\left(g_{i}\right)^{-1} f g_{i}$ sends $z_{i}$ onto $h\left(z_{i}\right)$ and fixes $\Delta \backslash\left\{z_{i}\right\}$. Composing this element with an element of $G_{\Delta}$, we find an element $f_{i}$, generated by $f$ and $G_{\Delta}$, which sends $z_{i}$ onto $h\left(z_{i}\right)$ and fixes $(\Delta \cup h(\Delta)) \backslash\left\{z_{i}, h\left(z_{i}\right)\right\}$.

Since $h^{-1} f_{1} \cdots f_{j}$ belongs to $G_{\Delta}$, this completes the proof.

Corollary 3.13. Assume that the ground field $\mathbf{k}$ is not a finite field of characteristic two and let $\Delta \subset \mathbb{A}^{n}(\mathbf{k})$ be a finite proper non-empty set, with $n \geqslant 2$. Then, $\operatorname{Aut}\left(\mathbb{A}^{n}, \Delta\right)$ is a maximal subgroup of $\operatorname{Aut}\left(\mathbb{A}^{n}\right)$.

Proof. If $\mathbf{k}$ is infinite, we use Lemma 3.12 and the fact that $\operatorname{Aut}\left(\mathbb{A}^{2}\right)$ acts $m$-transitively on $\mathbb{A}^{2}(\mathbf{k})$ for every $m \geqslant 1$, which can be seen using the subgroup

$$
\left\{\left(x_{1}, \ldots, x_{n}\right) \mapsto\left(x_{1}+p\left(x_{2}, \ldots, x_{n}\right), x_{2}, \ldots, x_{n}\right) \mid p \in \mathbf{k}\left[x_{2}, \ldots, x_{n}\right]\right\},
$$

and permutations of coordinates.

If $\mathbf{k}$ is a finite field of characteristic greater than two, the group $\operatorname{Aut}\left(\mathbb{A}^{2}\right)$ acts $m$-transitively on $\mathbb{A}^{2}(\mathbf{k})$ for each $m$ by [Mau01]; we can then apply Lemma 3.12 to $\Delta$ or its complement.

Corollary 3.14. Assume that the ground field $\mathbf{k}$ is a finite field of characteristic two and let $\Delta \subset \mathbb{A}^{n}(\mathbf{k})$ be a finite proper non-empty set, with $n \geqslant 2$. Then, $\operatorname{Aut}\left(\mathbb{A}^{n}, \Delta\right)$ is a maximal subgroup of $\operatorname{Aut}\left(\mathbb{A}^{n}\right)$ if and only if $|\Delta| \neq \frac{1}{2}\left|\mathbb{A}^{n}(\mathbf{k})\right|$.

Proof. Let us write $\left|\mathbb{A}^{n}(\mathbf{k})\right|=2 m$ for some integer $m$.

If $|\Delta|<m$, the fact that $\operatorname{Aut}\left(\mathbb{A}^{n}, \Delta\right)$ is a maximal subgroup of $\operatorname{Aut}\left(\mathbb{A}^{n}\right)$ follows from Lemma 3.12 and from the fact that the action of $\operatorname{Aut}\left(\mathbb{A}^{n}\right)$ on the $2 m$ points of $\mathbb{A}^{n}(\mathbf{k})$ give all even permutations (see [Mau01]), and thus acts $(2 m-2)$-transitively. If $|\Delta|>m$, we exchange $\Delta$ with its complement.

If $|\Delta|=m$, there exists an automorphism $\varphi$ of $\mathbb{A}^{n}$ exchanging $\Delta$ with its complement (by the result of [Mau01] cited before). Denoting by $H$ the group generated by $\operatorname{Aut}\left(\mathbb{A}^{n}, \Delta\right)$ and $\varphi$, we have $\operatorname{Aut}\left(\mathbb{A}^{n}, \Delta\right) \subsetneq H \subsetneq \operatorname{Aut}\left(\mathbb{A}^{n}\right)$.

Remark 3.15. Corollaries 3.13 and 3.14 raise the question of describing all maximal subgroups of $\operatorname{Aut}\left(\mathbb{A}^{n}\right)$ in general.

\subsection{Generalisation to higher dimension}

Let us show with an example that in higher dimension, the hypersurfaces $X \subset \mathbb{A}^{n}$ such that $\operatorname{Aut}\left(\mathbb{A}^{n}, X\right)$ is not an algebraic group are not as simple as in dimension $n=2$.

EXAMPLE 3.16. Let $X \subset \mathbb{A}^{3}$ be the hypersurface with equation $x y=f(z)$, for some polynomial $f \in \mathbf{k}[z]$. Then, $\operatorname{Aut}\left(\mathbb{A}^{3}, X\right)$ contains the group

$$
\left\{(x, y, z) \mapsto\left(x, y+\frac{f(z+x q(x))-f(z)}{x}, z+x q(x)\right) \mid q \in \mathbf{k}[x]\right\} \simeq \mathbf{k}[x]
$$




\section{Automorphisms of the plane PRESERVing a CuRVE}

and thus it is not an algebraic group.

A possible generalisation of Theorem 1 would be to show that every hypersurface $X \subset \mathbb{A}^{3}$ such that $\operatorname{Aut}\left(\mathbb{A}^{3}, X\right)$ is not an algebraic group admits an $\mathbb{A}^{1}$-fibration $X \rightarrow \mathbb{A}^{1}$ given by a coordinate projection.

\section{Classification of the possible group actions and the proof of Theorem 2}

This section is devoted to the proof of Theorem 2, which describes more precisely the curves and groups appearing in Theorem 1 , in the case where the ground field $\mathbf{k}$ is perfect and the curve is geometrically irreducible.

\subsection{The possibilities for $\Gamma$ and $\operatorname{Aut}\left(\mathbb{A}^{2}, \Gamma\right)$}

LEMma 4.1. Let $\Gamma$ be an affine geometrically irreducible curve, defined over a perfect field $\mathbf{k}$. The group $\operatorname{Aut}(\Gamma)$ is an affine algebraic group. If it has positive dimension, one of the following holds:

(i) $\Gamma \simeq \mathbb{A}^{1}$.

(ii) The curve $\Gamma$ is unicuspidal with normalisation $\mathbb{A}^{1}$.

(iii) Over the algebraic closure $\overline{\mathbf{k}}, \Gamma$ is isomorphic to $\mathbb{A}^{1} \backslash\{0\}$.

Proof. Let $\tilde{\Gamma}$ be the normalisation of $\Gamma$, which can be viewed as an open subset of a smooth projective curve $X$, defined over $\mathbf{k}$. Let $(X \backslash \tilde{\Gamma})(\overline{\mathbf{k}})=\left\{x_{1}, \ldots, x_{r}\right\}$ be its complement. The points $x_{1}, \ldots, x_{r}$ are not necessarily all defined over $\mathbf{k}$ (however, the union is invariant by the Galois $\operatorname{group} \operatorname{Gal}(\overline{\mathbf{k}} / \mathbf{k}))$. Moreover, we denote by $x_{r+1}, \ldots, x_{m}$ the $\overline{\mathbf{k}}$-points of $\tilde{\Gamma}$ which are send onto the singular $\overline{\mathbf{k}}$-points of $\Gamma$. As before, not all are necessarily defined over $\mathbf{k}$.

This yields a natural inclusion

$$
\operatorname{Aut}(\Gamma) \subseteq\left\{g \in \operatorname{Aut}(X) \mid g\left(\left\{x_{1}, \ldots, x_{m}\right\}\right)=\left\{x_{1}, \ldots, x_{m}\right\}\right\}
$$

and a group homomorphism $\operatorname{Aut}(\Gamma) \rightarrow \mathrm{Sym}_{m}$. The kernel is of the same dimension as $\operatorname{Aut}(\Gamma)$.

Let us recall some classic facts on automorphisms of smooth projective curves. If the genus of $X$ is at least two, then $\operatorname{Aut}(X)$ is finite. If the genus is one, the subgroup of $\operatorname{Aut}(X)$ that fixes a point is also finite. If $X$ is rational, the subgroup of automorphisms fixing three points is trivial.

- If the dimension of $\operatorname{Aut}(X)$ is positive, we obtain that $X$ is rational and $1 \leqslant r \leqslant m \leqslant 2$.

- If $r=m=1$, then $\Gamma=\tilde{\Gamma} \simeq \mathbb{A}^{1}$ (every form of the affine line over a perfect field is trivial; see [Rus70]).

- If $r=1$ and $m=2$, then $\tilde{\Gamma} \simeq \mathbb{A}^{1}$ and $\Gamma$ is a unicuspidal curve.

- If $r=m=2$, then $\Gamma=\tilde{\Gamma}$ is smooth and isomorphic, over $\overline{\mathbf{k}}$, to $\mathbb{A}^{1} \backslash\{0\}$.

Remark 4.2. Lemma 4.1 is false over a non-perfect field, since there are non-trivial forms of the affine line and its additive group; see [Rus70] for a classification of such curves.

Lemma 4.3. Assume that $\mathbf{k}$ is a perfect field. Let $\Gamma \subseteq \mathbb{A}^{2}$ be a closed geometrically irreducible curve and assume that Aut $\left(\mathbb{A}^{2}, \Gamma\right)$ is an algebraic group of positive dimension (over $\left.\overline{\mathbf{k}}\right)$. Then, $\operatorname{Aut}\left(\mathbb{A}^{2}, \Gamma\right)$ contains a closed subgroup $G$ defined over $\mathbf{k}$, which is either

(i) a one-dimensional torus, that is, isomorphic to the one-dimensional multiplicative group $\mathbb{G}_{m}$ over $\overline{\mathbf{k}}$, or 


\section{JÉRÉMY BLANC AND IMMANUEl StAMPfli}

(ii) isomorphic to the one-dimensional additive group $\mathbb{G}_{a}$ over $\mathbf{k}$.

Proof. The algebraic group $\operatorname{Aut}\left(\mathbb{A}^{2}, \Gamma\right)$ is isomorphic to a closed subgroup of $\operatorname{Aut}(\Gamma)$ (Theorem 1$)$. This gives three possibilities for $\Gamma$, according to Lemma 4.1.

(a) We have $\Gamma \simeq \mathbb{A}^{1}$, and therefore $\operatorname{Aut}(\Gamma) \simeq \mathbb{G}_{a} \rtimes \mathbb{G}_{m}$.

(b) The curve $\Gamma$ is unicuspidal, in which case $\operatorname{Aut}(\Gamma)$ is a torus.

(c) The curve $\Gamma$ is isomorphic to $\mathbb{A}^{1} \backslash\{0\}$ over $\overline{\mathbf{k}}$. The connected component of the identity $\operatorname{Aut}\left(\mathbb{A}^{2}, \Gamma\right)^{0}$ is a connected algebraic group, defined over $\mathbf{k}$, which is a torus.

The two possibilities given by Lemma 4.3 are described in $\S 4.2$ and $\S 4.3$, respectively. We will, in particular, show that the second case does not occur.

\subsection{Torus actions}

Lemma 4.4. Assume that $\mathbf{k}$ is a perfect field. Let $\Gamma \subseteq \mathbb{A}^{2}$ be a closed geometrically irreducible curve and assume that $\operatorname{Aut}\left(\mathbb{A}^{2}, \Gamma\right)$ is an algebraic group that contains a closed one-dimensional torus $T$. Then, there exists an automorphism $\varphi: \mathbb{A}^{2} \rightarrow \mathbb{A}^{2}$ such that $\varphi \circ T \circ \varphi^{-1} \subseteq \operatorname{GL}(2, \mathbf{k})$.

Proof. By Corollary 3.9, we can assume that we have either $\operatorname{Aut}\left(\mathbb{A}^{2}, \Gamma\right) \subseteq \operatorname{Aut}\left(\mathbb{F}_{n}, E_{n} \cup L_{\mathbb{F}_{n}}\right)$ or $\operatorname{Aut}\left(\mathbb{A}^{2}, \Gamma\right) \subseteq \operatorname{Aut}\left(\mathbb{P}^{2}, L_{\mathbb{P}^{2}}\right)$.

Moreover, we can assume that $T$ is defined over $\mathbf{k}$ and $T$ is isomorphic to $\mathbb{G}_{m}$ over $\overline{\mathbf{k}}$ (Lemma 4.3).

Assume $\operatorname{Aut}\left(\mathbb{A}^{2}, \Gamma\right) \subseteq \operatorname{Aut}\left(\mathbb{F}_{n}, E_{n} \cup L_{\mathbb{F}_{n}}\right)$. Thus $T$ acts on $L_{\mathbb{F}_{n}}$. If this action is trivial, then there exists a fixed point on $L_{\mathbb{F}_{n}} \backslash E_{n}$ that is defined over $\mathbf{k}$. Otherwise, $T$ has exactly two fixed points on $L_{\mathbb{F}_{n}} \simeq \mathbb{P}^{1}$ defined over $\overline{\mathbf{k}}$. As $L_{\mathbb{F}_{n}} \cap E_{n}$ is a fixed point of the $T$-action that is defined over $\mathbf{k}$, there is a fixed point on $L_{\mathbb{F}_{n}} \backslash E_{n}$ that is defined over $\mathbf{k}$. Thus by performing elementary links, we can assume that $T \subseteq \operatorname{Aut}\left(\mathbb{F}_{1}, E_{1} \cup L_{\mathbb{F}_{1}}\right)$. But $T$ preserves the exceptional divisor $E_{1}$ and therefore $\varphi \circ T \circ \varphi^{-1} \subseteq \operatorname{Aut}\left(\mathbb{P}^{2}, L_{\mathbb{P}^{2}}\right)$, where $\varphi: \mathbb{F}_{1} \rightarrow \mathbb{P}^{2}$ denotes a link of type III.

Thus we are left with the case $T \subseteq \operatorname{Aut}\left(\mathbb{P}^{2}, L_{\mathbb{P}^{2}}\right)$. It is enough to show that the induced action of $T$ on $\mathbb{A}^{2}=\mathbb{P}^{2} \backslash L_{\mathbb{P}^{2}}$ has a fixed point that is defined over $\mathbf{k}$. The set of points of $\mathbb{A}^{2}(\overline{\mathbf{k}})$ that are fixed by $T(\overline{\mathbf{k}})$ consists of either one affine line or one point. This set is invariant by the action of the Galois group $\operatorname{Gal}(\overline{\mathbf{k}} / \mathbf{k})$, and is thus defined over $\mathbf{k}$. Using again the fact that every form of the affine line over a perfect field is trivial (see [Rus70]), we find a k-point of $\mathbb{A}^{2}$ fixed by $T$.

Proposition 4.5. Assume that $\mathbf{k}$ is perfect. Let $\Gamma \subseteq \mathbb{A}^{2}$ be a closed geometrically irreducible curve and assume that $\operatorname{Aut}\left(\mathbb{A}^{2}, \Gamma\right)$ contains a closed one-dimensional torus. After conjugation by an automorphism of $\mathbb{A}^{2}$, the curve $\Gamma$

(i) has equation $x=0$, or

(ii) has equation $x^{b}=\lambda y^{a}$, where $a, b>1$ are coprime integers and $\lambda \in \mathbf{k}^{*}$, or

(iii) has equation $x^{b} y^{a}=\lambda$, where $a, b \geqslant 1$ are coprime integers and $\lambda \in \mathbf{k}^{*}$, or

(iv) has equation $\lambda x^{2}+\nu y^{2}=1$, where $\lambda, \nu \in \mathbf{k}^{*},-\lambda \nu$ is not a square in $\mathbf{k}$ and char $(\mathbf{k}) \neq 2$, or

(v) has equation $x^{2}+\mu x y+y^{2}=1$, where $\mu \in \mathbf{k}^{*}, x^{2}+\mu x+1$ has no root in $\mathbf{k}$ and $\operatorname{char}(\mathbf{k})=2$.

Moreover, the group $\operatorname{Aut}\left(\mathbb{A}^{2}, \Gamma\right)$ is, respectively, equal to

(i) $\left\{(x, y) \mapsto(a x, b y+P(x)) \mid a, b \in \mathbf{k}^{*}, P \in \mathbf{k}[x]\right\} \simeq \mathbf{k}[x] \rtimes\left(\mathbf{k}^{*}\right)^{2}$,

(ii) $\left\{(x, y) \mapsto\left(t^{a} x, t^{b} y\right) \mid t \in \mathbf{k}^{*}\right\} \simeq \mathbf{k}^{*}$, 


\section{Automorphisms of the Plane PRESERVING A CURVE}

(iii) $\left\{(x, y) \mapsto\left(t^{a} x, t^{-b} y\right) \mid t \in \mathbf{k}^{*}\right\} \simeq \mathbf{k}^{*}$ if $(a, b) \neq(1,1)$, $\left\{(x, y) \mapsto\left(t x, t^{-1} y\right) \mid t \in \mathbf{k}^{*}\right\} \cup\left\{(x, y) \mapsto\left(t y, t^{-1} x\right) \mid t \in \mathbf{k}^{*}\right\} \simeq \mathbf{k}^{*} \rtimes \mathbb{Z} / 2 \mathbb{Z}$ if $(a, b)=(1,1)$,

(iv) $T \rtimes\langle\sigma\rangle \simeq T \rtimes \mathbb{Z} / 2 \mathbb{Z}$, where $T,\{\sigma\} \subset \mathrm{GL}(2, \mathbf{k})$ are given by

$$
T=\left\{\left(\begin{array}{cc}
a & -\nu b \\
\lambda b & a
\end{array}\right) \mid a^{2}+\lambda \nu b^{2}=1\right\}, \quad \sigma=\left(\begin{array}{cc}
1 & 0 \\
0 & -1
\end{array}\right)
$$

and $T$ is a torus, which is not $\mathbf{k}$-split,

(v) $T \rtimes\langle\sigma\rangle \simeq T \rtimes \mathbb{Z} / 2 \mathbb{Z}$, where $T,\{\sigma\} \subset \mathrm{GL}(2, \mathbf{k})$ are given by

$$
T=\left\{\left(\begin{array}{cc}
a & b \\
b & a+\mu b
\end{array}\right) \mid a^{2}+\mu a b+b^{2}=1\right\}, \quad \sigma=\left(\begin{array}{cc}
1 & \mu \\
0 & 1
\end{array}\right)
$$

and $T$ is a torus which is isomorphic to $\Gamma$ (and which is not $\mathbf{k}$-split).

Proof. By Theorem 1, we can suppose that $\operatorname{Aut}\left(\mathbb{A}^{2}, \Gamma\right)$ is an algebraic group, and using Lemma 4.4, we can moreover suppose that $\Gamma$ is preserved by a torus $T \subset \mathrm{GL}(2, \mathbf{k})$.

There exists an element $\psi \in \mathrm{GL}(2, \overline{\mathbf{k}})$ which conjugates $T(\overline{\mathbf{k}})$ to

$$
\lambda \mapsto\left(\begin{array}{cc}
\lambda^{a} & 0 \\
0 & \lambda^{b}
\end{array}\right)
$$

for integers $a, b$ with $(a, b) \neq(0,0)$ and $a, b$ coprime. If $a$ or $b$ is equal to zero, then it follows that $\Gamma$ is a line. Hence, we can assume that $a$ and $b$ are non-zero. Now, let $\left(x_{0}, y_{0}\right) \in \psi(\Gamma(\overline{\mathbf{k}}))$ such that $\left(x_{0}, y_{0}\right) \neq(0,0)$. If $x_{0}$ or $y_{0}$ is zero, then $\Gamma$ is again a line. Hence, we may assume that $x_{0} \neq 0 \neq y_{0}$. By symmetry, we can assume that $b>0$. Then, the equation of $\psi(\Gamma)$ is

$$
\begin{array}{ll}
y_{0}^{a} x^{b}-x_{0}^{b} y^{a}=0 & \text { if } a>0, \\
y_{0}^{a} x^{b} y^{-a}-x_{0}^{b}=0 & \text { if } a<0 .
\end{array}
$$

In the first case, we can assume that $a>1$ or $b>1$, otherwise the curve is a line. This implies that $a \neq b$, since the two are coprime, and we can thus assume that $a>b$. The Galois group $\operatorname{Gal}(\overline{\mathbf{k}} / \mathbf{k})$ fixes the unique point at infinity, which is then defined over $\mathbf{k}$. Hence, we can assume that the unique point of the closure of $\Gamma$ at infinity is the direction $y=0$, and that the equation of $\Gamma$ is the polynomial $(\alpha x+\beta y)^{b}-(\gamma y)^{a} \in \mathbf{k}[x, y]$, for some $\alpha, \beta, \gamma \in \overline{\mathbf{k}}, \alpha \gamma \neq 0$. If $\beta / \alpha \in \mathbf{k}$, we make a change of coordinates $(x, y) \mapsto(x-(\beta / \alpha) y, y)$ and obtain an equation of the form $x^{b}-\lambda y^{a}$ for some $\lambda \in \mathbf{k}$, as desired. It remains to see that $\beta / \alpha$ always belongs to $\mathbf{k}$. If the characteristic of $\mathbf{k}$ is zero, we develop $(\alpha x+\beta y)^{b}$ and divide the coefficient of $x^{b-1} y$ by the coefficient of $x^{b}$. If the characteristic of $\mathbf{k}$ is $p>0$, we write $b=q m$, where $q$ is a power of $p$ and $p$ does not divide $m$. Developing, we find

$$
(\alpha x+\beta y)^{b}=\left(\alpha^{q} x^{q}+\beta^{q} y^{q}\right)^{m}=\alpha^{b} x^{b}+m \alpha^{b-q} \beta^{q} x^{b-q} y^{q}+\ldots
$$

hence $(\beta / \alpha)^{q} \in \mathbf{k}$, which implies, since $\mathbf{k}$ is a perfect field, that $\beta / \alpha \in \mathbf{k}$.

In the second case $(a<0)$, the closure of the curve $\psi(\Gamma)$ has two points at infinity in $\mathbb{P}^{2}$. If $a \neq-b$, the two points have different multiplicities. Consequently, the Galois group $\operatorname{Gal}(\overline{\mathbf{k}} / \mathbf{k})$ has to fix the two points at infinity of $\Gamma$, which are thus defined over $\mathbf{k}$. We can assume that these points correspond to the directions $x=0$ and $y=0$, and that $\psi \in \operatorname{GL}(2, \overline{\mathbf{k}})$ is diagonal. The equation of $\Gamma$ is then of the form $x^{b} y^{a}-\lambda$ for some $\lambda \in \mathbf{k}^{*}$, and we get case (iii). The only remaining case is when $(-a, b)=(1,1)$ and the two points at infinity of $\Gamma$ are exchanged by $\operatorname{Gal}(\overline{\mathbf{k}} / \mathbf{k})$. The equation of $\psi(\Gamma)$ being of the form $x y=x_{0} y_{0}$, the equation of $\Gamma$ is of the form

$$
\lambda x^{2}+\mu x y+\nu y^{2}=1,
$$




\section{JÉRÉMy BlanC AND IMMANUEl StAMPfli}

where $\lambda, \mu, \nu \in \mathbf{k}$, and $\lambda x^{2}+\mu x y+\nu y^{2} \in \mathbf{k}[x, y]$ is irreducible. When the characteristic of $\mathbf{k}$ is not two, we can make a change of coordinates $(x, y) \mapsto(x-(\mu / 2 \lambda) y, y)$ and assume $\mu=0$. The two points at infinity are thus given by $\lambda x^{2}+\nu y^{2}=0$. Because the two points are not defined over $\mathbf{k}$, we find that $-\nu \lambda$ is not a square in $\mathbf{k}$, and obtain (iv). If the characteristic of $\mathbf{k}$ is two, the elements $\lambda, \nu$ are squares in $\mathbf{k}$, since $\mathbf{k}$ is perfect. Making a diagonal change of variables, we can then assume $\lambda=\nu=1$. Then $x^{2}+\mu x+1$ has no root in $\mathbf{k}$, and we get case (v).

It remains to prove that $\operatorname{Aut}\left(\mathbb{A}^{2}, \Gamma\right)$ has the desired form. Case (i) is a direct calculation. In cases (ii)-(v), it can be checked that the given group is contained in $\operatorname{Aut}\left(\mathbb{A}^{2}, \Gamma\right)$, so we just need to see that there is no other automorphism. Since the group homomorphism $\operatorname{Aut}\left(\mathbb{A}^{2}, \Gamma\right) \rightarrow \operatorname{Aut}(\Gamma)$ is injective, the only case to consider is the curve $x^{b} y^{a}-\lambda=0$, with $(a, b) \neq(1,1)$, and to prove that there is no automorphism of $\mathbb{A}^{2}$ inducing on $\Gamma$ an "exchange" of the two points at infinity. These two points are $p_{1}=(1: 0: 0) \in \mathbb{P}^{2}$ and $p_{2}=(0: 1: 0) \in \mathbb{P}^{2}$, and have multiplicity $a$ and $b$, respectively, on $\Gamma$, and $\operatorname{ht}_{\Gamma}\left(p_{1}\right)=\operatorname{ht}_{\Gamma}\left(p_{2}\right)>0$ if $b>1$. We can moreover assume $a>b \geqslant 1$. The hypothetical automorphism extends to a birational map $\varphi: \mathbb{P}^{2}-\rightarrow \mathbb{P}^{2}$ which is not an automorphism of $\mathbb{P}^{2}$, and thus decomposes into a sequence of elementary links $\varphi=\varphi_{m} \cdots \varphi_{1}$ (Proposition 2.10). By Corollary 3.5, we have $b=1$ and the point blown up by the first link $\varphi_{1}:\left(\mathbb{P}^{2}, L_{\mathbb{P}^{2}}\right) \rightarrow\left(\mathbb{F}_{1}, E_{1} \cup L_{\mathbb{F}_{1}}\right)$ is $p_{1}$. Looking at the equation of $\Gamma$ in $\mathbb{A}^{2}$, we can describe the closure of $\Gamma$ on $\mathbb{F}_{1}$. This projective curve is smooth, intersects transversally $L_{\mathbb{F}_{1}}$ in one point away from $E_{1}$, corresponding to $p_{2}$, and intersects $E_{1}$ in one point $q_{1}$, with multiplicity $a$, corresponding to $p_{1}$; this latter is moreover not on $L_{\mathbb{F}_{1}}$. Consequently, the next links of type II do not affect the point $q_{1}$, and after the first link of type III, the image of the curve is singular at a point of $\mathbb{P}^{2}$ with multiplicity at least $a$ and height one. Since this point is not the base point of the next elementary links, the image of $\Gamma$ by $\varphi$ again has a singular point, corresponding to the image of $p_{1}$. It is thus not possible to "exchange" $p_{1}$ and $p_{2}$.

\section{$4.3 \mathbb{G}_{a}$-actions}

The classification of all $\mathbb{G}_{a}$-actions on $\mathbb{A}^{2}$ is known when the ground field $\mathbf{k}$ is of characteristic zero [Ren68] or algebraically closed of positive characteristic [Miy71]. The following lemma gives the generalisation of Miyanishi's result to the case where $\mathbf{k}$ is perfect. The proof is probably known to the specialists, we include it for the sake of completeness and lack of reference.

Proposition 4.6. Assume that $\mathbf{k}$ is a perfect field of characteristic $p>0$. Then every $\mathbb{G}_{a}$-action on $\mathbb{A}^{2}$ that is defined over $\mathbf{k}$ has the form

$$
(t, x, y) \mapsto\left(x, y+t f_{0}(x)+t^{p} f_{1}(x)+\ldots+t^{p^{n}} f_{n}(x)\right)
$$

up to conjugation with a $\mathbf{k}$-automorphism of $\mathbb{A}^{2}$.

Proof. We fix a non-trivial $\mathbb{G}_{a}$-action on $\mathbb{A}^{2}$ that is defined over $\mathbf{k}$. By [Miy71] it follows that the $\mathbb{G}_{a}$-action has the claimed form over the algebraic closure $\overline{\mathbf{k}}$ (up to conjugation with a $\overline{\mathbf{k}}$ automorphism of $\mathbb{A}^{2}$ ). Thus there exists a $\mathbb{G}_{a}$-invariant polynomial $f \in \overline{\mathbf{k}}[x, y]$ which is a variable of $\overline{\mathbf{k}}[x, y]$, that is, which admits a $g \in \overline{\mathbf{k}}[x, y]$ such that $\overline{\mathbf{k}}[f, g]=\overline{\mathbf{k}}[x, y]$. Let $G:=\operatorname{Gal}(\overline{\mathbf{k}} / \mathbf{k})$ be the Galois group. Now, $\overline{\mathbf{k}}[f]$ is the subring of $\mathbb{G}_{a}$-invariant polynomials of $\overline{\mathbf{k}}[x, y]$ and since the $\mathbb{G}_{a}$-action is defined over $\mathbf{k}$, the subspace $\overline{\mathbf{k}}[f]$ is invariant under $G$. Thus, the action of $G$ on $\overline{\mathbf{k}}[f, g]=\overline{\mathbf{k}}[x, y]$ is given by

$$
\sigma(f)=a_{\sigma} f+c_{\sigma}, \quad \sigma(g)=b_{\sigma} g+d_{\sigma},
$$

where $a_{\sigma}, b_{\sigma} \in \overline{\mathbf{k}}^{*}, c_{\sigma} \in \overline{\mathbf{k}}$ and $d_{\sigma} \in \overline{\mathbf{k}}[f]$. It is enough to show that the 1-cocycle

$$
G \rightarrow \mathrm{J}_{n}(\overline{\mathbf{k}}) \simeq\left(\overline{\mathbf{k}}^{*}\right)^{2} \ltimes(\overline{\mathbf{k}} \ltimes \overline{\mathbf{k}}[f]), \quad \sigma \mapsto\left(a_{\sigma}, b_{\sigma}, c_{\sigma}, d_{\sigma}\right)
$$




\section{Automorphisms of the plane PRESERVing a CuRVE}

is a 1-coboundary. The vanishing of $H^{1}\left(G, J_{n}(\overline{\mathbf{k}})\right)$ follows from the vanishing of $H^{1}\left(G, \overline{\mathbf{k}}^{*}\right)$ (see [NSW00, (6.2.1) Theorem]) and from that of $H^{1}(G, \overline{\mathbf{k}}[f])=\underline{\lim }_{n} H^{1}(G, \overline{\mathbf{k}}[f] \leqslant n$ ) (see [NSW00, (1.5.1) Proposition] and [NSW00, (6.1.1) Theorem]) by using exact sequences (here $\overline{\mathbf{k}}[f]_{\leqslant n}$ denotes the polynomials in $f$ of degree at $\operatorname{most} n$ ).

Lemma 4.7. Assume that $\mathbf{k}$ is perfect. Let $\Gamma \subseteq \mathbb{A}^{2}$ be a closed geometrically irreducible curve that is defined over $\mathbf{k}$ and assume that it is preserved under a non-trivial $\mathbb{G}_{a}$-action (defined over $\mathbf{k})$. Then, there exists an automorphism $\varphi: \mathbb{A}^{2} \rightarrow \mathbb{A}^{2}$ such that $\varphi(\Gamma)$ is an affine line in $\mathbb{A}^{2}$.

Proof. By Proposition 4.6 (in the case $\operatorname{char}(\mathbf{k})=p>0$ ) and by [Ren68] (in the case char $(\mathbf{k})=0$ ) we can conjugate the action to an action of the form $(t, x, y) \mapsto(x, y+p(t, x))$, where $p \in \mathbf{k}[t, x]$ is a non-zero polynomial. Hence, every geometrically irreducible $\mathbb{G}_{a}$-invariant curve is a line in $\mathbb{A}^{2}$.

Lemma 4.7 implies that the second case of Lemma 4.3 does not occur. The proof of Theorem 2 is now clear.

Proof of Theorem 2. By Theorem 1, either $\Gamma$ is a line or $\operatorname{Aut}\left(\mathbb{A}^{2}, \Gamma\right)$ is an algebraic subgroup of $\operatorname{Aff}\left(\mathbb{A}^{2}\right)$ or $\mathrm{J}_{n}$ for some $n \geqslant 1$. If $\operatorname{Aut}\left(\mathbb{A}^{2}, \Gamma\right)$ is an algebraic group of positive dimension, it contains a closed one-dimensional torus by Lemmas 4.3 and 4.7. The description of the possible cases then follows from Proposition 4.5.

\subsection{The case of finite groups}

There are many examples where $\operatorname{Aut}\left(\mathbb{A}^{2}, \Gamma\right)$ is finite. The simplest way to get such examples is to take a finite subgroup $G \subset \operatorname{Aut}\left(\mathbb{A}^{2}\right)$ and to look for invariant curves. Since $G$ has a finite action on $\mathbf{k}[x, y]$, one can find many invariant polynomials, and in practice most of them are irreducible.

In characteristic zero, the group $G$ is reductive and thus contained, up to conjugation, in $\mathrm{GL}_{2}$ (see [Kam79]). In positive characteristic, there are, however, many non-linearisable subgroups of $\operatorname{Aut}\left(\mathbb{A}^{2}\right)$, and, as far as we know, there is for the moment no classification of the conjugacy classes of such subgroups.

EXAMPLE 4.8. Let $\mathbf{k}$ be of characteristic $p>0$. For any integer $a>1$,

$$
\varphi:(x, y) \mapsto\left(x, y+x^{a}\right)
$$

is a non-linearisable automorphism of order $p$ of $\mathbb{A}^{2}$, which preserves the family of curves of the form

$$
y^{p}-y x^{a(p-1)}=q(x),
$$

where $q \in \mathbf{k}[x]$.

\section{ACKNOWLEDGEMENTS}

We would like to thank the referee for his helpful remarks and corrections.

\section{REFERENCES}

AM75 S. Abhyankar and T.T. Moh, Embeddings of the line in the plane, J. Reine Angew. Math. 276 (1975), 148-166. 


\section{JÉRÉMy BLANC AND IMMANUEl StAMPfli}

AZ13 I. Arzhantsev and M. Zaidenberg, Acyclic curves and group actions on affine toric surfaces, in Affine algebraic geometry, 1-41, World Sci. Publ., Hackensack, NJ, 2013. http://dx.doi. org/10.1142/9789814436700_0001

BD11 J. Blanc and A. Dubouloz, Automorphisms of $\mathbb{A}^{1}$-fibered affine surfaces, Trans. Amer. Math. Soc. 363 (2011), no. 11, 5887-5924. http://dx.doi.org/10.1090/ S0002-9947-2011-05266-9

BŻa10 M. Borodzik and H. Żoładek, Complex algebraic plane curves via Poincaré-Hopf formula II: Annuli, Israel J. Math. 175 (2010), 301-347. http://dx.doi .org/10.1007/s11856-010-0013-1

CNKR09 P. Cassou-Noguès, M. Korás and P. Russell, Closed embeddings of $\mathbb{C}^{*}$ in $\mathbb{C}^{2}$. I, J. Algebra 322 (2009), no. 9, 2950-3002. http://dx.doi.org/10.1016/j.jalgebra.2008.11.013

Cor95 A. Corti, Factoring birational maps of threefolds after Sarkisov, J. Algebraic Geom. 4 (1995), no. 2, 223-254.

FM89 S. Friedland and J. Milnor, Dynamical properties of plane polynomial automorphisms, Ergod. Th \& Dyn. Syst. 9 (1989), 67-99.

Isk96 V.A. Iskovskikh, Factorization of birational mappings of rational surfaces from the point of view of Mori theory, Russian Math. Surveys 51 (1996), no. 4, 585-652.

Jel03 Z. Jelonek, The set of fixed points of an algebraic group, Bull. Polish Acad. Sci. Math. 51 (2003), no. 1, 69-73.

Jun42 H.W.E. Jung, Über ganze birationale Transformationen der Ebene, J. Reine Angew. Math. 184 (1942), 161-174.

Kam79 T. Kambayashi, Automorphism group of a polynomial ring and algebraic group action on an affine space, J. Algebra 60 (1979), 439-451.

KPR14 M. Koras, K. Palka and P. Russell, The geometry of sporadic $\mathbb{C}^{*}$-embeddings into $\mathbb{C}^{2}$, arXiv: 1405.6872.

vdK53 W. van der Kulk, On polynomial rings in two variables, Nieuw Arch. Wisk. 1 (1953), 33-41.

Kum02 S. Kumar, Kac-Moody groups, their flag varieties and representation theory, Progress in Mathematics, 204, Birkhaüser Boston, Inc., Boston, MA, 2002. http://dx.doi.org/10.1007/ 978-1-4612-0105-2

Lam02 S. Lamy, Une preuve géométrique du théorème de Jung, Enseign. Math. (2) 48 (2002), no. 3-4, 291-315. http://dx.doi.org/10.5169/seals-66078

Mau01 S. Maubach, Polynomial automorphisms over finite fields, Serdica Math. J. 27 (2001), no. 4, 343-350.

Miy71 M. Miyanishi, $G_{a}$-action of the affine plane, Nagoya Math. J. 41 (1971), 97-100.

Nag71 M. Nagata, A theorem of Gutwirth, J. Math. Kyoto Univ. 11 (1971), 149-154.

NSW00 J. Neukirch, A. Schmidt and K. Wingberg, Cohomology of number fields, Grundlehren der Mathematischen Wissenschaften, Vol. 323, Springer-Verlag, Berlin, 2000. http://dx.doi .org/ 10.1007/978-3-540-37889-1

Ren68 R. Rentschler, Opérations du groupe additif sur le plan affine, C. R. Acad. Sci. Paris Sér. A-B 267 (1968), A384-A387.

Rus70 P. Russell, Forms of the affine line and its additive group, Pacific J. Math. 32 (1970), no. 2, $527-539$.

Suz74 M. Suzuki, Propriétés topologiques des polynômes de deux variables complexes, et automorphismes algbriques de l'espace $\mathbb{C}^{2}$, J. Math. Soc. Japan 26 (1974), 241-257.

Wri79 D. Wright, Abelian subgroups of $\operatorname{Aut}_{k}(k[X, Y])$ and applications to actions on the affine plane, Illinois J. Math. 23 (1979), no. 4, 579-634.

ZL83 M. Zaidenberg and V.Ya. Lin, An irreducible, simply connected algebraic curve in $\mathbf{C}^{2}$ is equivalent to a quasihomogeneous curve, Dokl. Akad. Nauk SSSR 271 (1983), no. 5, 1048-1052. 
Automorphisms of the Plane PRESERVing a CURVE

Jérémy Blanc Jeremy.Blanc@unibas.ch

Mathematisches Institut, Universität Basel, Rheinsprung 21, 4051 Basel, Switzerland

Immanuel Stampfli Immanuel.E.Stampfli@gmail.com

Mathematisches Institut, Universität Basel, Rheinsprung 21, 4051 Basel, Switzerland 